\title{
Structure-based directed evolution improves S. cerevisiae growth on xylose by influencing in vivo enzyme performance
}

\author{
Misun Lee ${ }^{1}$, Henriëtte J. Rozeboom ${ }^{1}$, Eline Keuning ${ }^{1}$, Paul de Waal ${ }^{2}$ and Dick B. Janssen ${ }^{1 *}$ (D)
}

\begin{abstract}
Background: Efficient bioethanol production from hemicellulose feedstocks by Saccharomyces cerevisiae requires xylose utilization. Whereas $S$. cerevisiae does not metabolize xylose, engineered strains that express xylose isomerase can metabolize xylose by converting it to xylulose. For this, the type II xylose isomerase from Piromyces (PirXI) is used but the in vivo activity is rather low and very high levels of the enzyme are needed for xylose metabolism. In this study, we explore the use of protein engineering and in vivo selection to improve the performance of PirXI. Recently solved crystal structures were used to focus mutagenesis efforts.

Results: We constructed focused mutant libraries of Piromyces xylose isomerase by substitution of second shell residues around the substrate- and metal-binding sites. Following library transfer to $S$. cerevisiae and selection for enhanced xylose-supported growth under aerobic and anaerobic conditions, two novel xylose isomerase mutants were obtained, which were purified and subjected to biochemical and structural analysis. Apart from a small difference in response to metal availability, neither the new mutants nor mutants described earlier showed significant changes in catalytic performance under various in vitro assay conditions. Yet, in vivo performance was clearly improved. The enzymes appeared to function suboptimally in vivo due to enzyme loading with calcium, which gives poor xylose conversion kinetics. The results show that better in vivo enzyme performance is poorly reflected in kinetic parameters for xylose isomerization determined in vitro with a single type of added metal.

Conclusion: This study shows that in vivo selection can identify xylose isomerase mutants with only minor changes in catalytic properties measured under standard conditions. Metal loading of xylose isomerase expressed in yeast is suboptimal and strongly influences kinetic properties. Metal uptake, distribution and binding to xylose isomerase are highly relevant for rapid xylose conversion and may be an important target for optimizing yeast xylose metabolism.
\end{abstract}

Keywords: Xylose isomerase, Metalloenzyme, Directed evolution, Bioethanol, Protein engineering

\section{Background}

Efficient xylose conversion is an important property when selecting or engineering yeast strains to be used in second-generation bioethanol production. Fermentation

\footnotetext{
*Correspondence: d.b.janssen@rug.nl

${ }^{1}$ Biochemical Laboratory, Groningen Biomolecular Sciences

and Biotechnology Institute, University of Groningen, Nijenborgh 4, 9747 AG Groningen, The Netherlands

Full list of author information is available at the end of the article
}

of lignocellulose-derived feedstocks, which contain up to $30 \%$ D-xylose, is often carried out by Saccharomyces cerevisiae. Since this yeast does not metabolize the aldopentose D-xylose naturally, incorporation of either xylose isomerase or a combination of xylose reductase and xylitol dehydrogenase is necessary to convert D-xylose to the ketose D-xylulose, which can be metabolized [14]. The use of xylose isomerase has the advantage over the xylose reductase-xylitol dehydrogenase system that there is no intermediate production of xylitol and less

c) The Author(s) 2020. This article is licensed under a Creative Commons Attribution 4.0 International License, which permits use, sharing, adaptation, distribution and reproduction in any medium or format, as long as you give appropriate credit to the original author(s) and the source, provide a link to the Creative Commons licence, and indicate if changes were made. The images or other third party material in this article are included in the article's Creative Commons licence, unless indicated otherwise in a credit line to the material. If material is not included in the article's Creative Commons licence and your intended use is not permitted by statutory regulation or exceeds the permitted use, you will need to obtain permission directly from the copyright holder. To view a copy of this licence, visit http://creativeco mmons.org/licenses/by/4.0/. The Creative Commons Public Domain Dedication waiver (http://creativecommons.org/publicdomain/ zero/1.0/) applies to the data made available in this article, unless otherwise stated in a credit line to the data. 
formation of side products, but combining the pathways may also have certain benefits [5]. Several efforts to find suitable xylose isomerases have been reported [6-11]. A xylose isomerase discovered by Kuyper et al. [12, 13] in the fungal strain Piromyces E2 through genome mining (PirXI) is an attractive candidate for xylose isomerization in engineered S. cerevisiae strains, and is used in several studies [14]. However, in vivo performance of the enzyme is modest, as indicated by the high copy number (up to 10) of the chromosomally inserted XI-encoding gene observed in evolved strains that are capable of anaerobic D-xylose fermentation [15]. A multi-copy plasmid leading to overproduction of the PirXI protein has also been used for enhanced xylose metabolism [16]. The engineering of yeast strains showing faster xylose metabolism is an important challenge in the pursuit of strain improvement for second-generation bioethanol production [17-20].

The observation that strains with multiple copies of PirXI genes evolve during prolonged adaptation suggests that in vivo enzyme activity in S. cerevisiae is limiting xylose turnover [21]. Mutations in different xylose isomerases can lead to accelerated xylose metabolism and protein engineering of xylose isomerase is receiving significant attention [11, 14, 19, 20]. However, it is unclear which properties of the enzyme need to be tailored to improve its in vivo performance. A straightforward hypothesis is that the kinetic properties as reflected in catalytic rate $\left(k_{\text {cat }}\right)$ and/or substrate affinity $\left(K_{\mathrm{M}}\right)$ at physiological conditions are not optimal for efficient xylose metabolism. On the other hand, metal affinity and in vivo metal content of xylose isomerase may also play an important role causing the enzyme to function suboptimally. Xylose isomerase is a metalloenzyme that requires two divalent metals for activity, and the wild type shows the best activity with $\mathrm{Mn}^{2+}$ [22]. However, metal content of the enzyme expressed in yeast may vary [21, 22]. A yeast strain with a mutation in its PMR1 gene which influences manganese homeostasis and increases $\mathrm{Mn}^{2+}$ content of the PirXI protein showed an enhanced rate of xylose consumption [21]. The high expression levels of the PirXI protein in selected xylose-metabolizing strains could be a burden for cell growth, and reduced expression of a more active enzyme would improve xylose consumption and growth rates. In view of the complexity of yeast cells, there may well be other factors that determine the performance of heterologously expressed enzymes, including compartmentalization, enzyme stability, and competition for metals with other cellular components.

Modern protein engineering tools enable tailoring of enzyme properties for specific applications. A particularly effective strategy is the use of directed evolution, i.e., the construction of mutant libraries and screening those for improved variants [23-25]. This approach does not require structural information. Here, one can take advantage of the fact that xylose isomerase activities limit the growth of S. cerevisiae on D-xylose and employ a random mutagenesis method with in vivo selection for improved growth $[9,14]$. This allowed the discovery of unexpected xylose isomerase mutations, some of which were far away from the active site. It is known that distant mutations can enhance activity, e.g., by influencing enzyme surface properties [26]. On the other hand, the lack of focus in random mutagenesis protocols yields libraries with a low abundance of beneficial mutations, and a very large number of mutants often must be screened to discover better enzymes. So-called smart libraries, which incorporate phylogenetic and structural information in the design, are assumed to better cover functional sequence space, increasing the chance of discovering useful mutations and reducing the need for extensive screening [27-29].

To support PirXI engineering and understand the effects of selected mutations, we have recently characterized the enzyme both structurally and biochemically [22]. Even though the unidentified causes of the modest in vivo performance of PirXI and the complexity of the kinetic mechanism cause uncertainty about the types of mutation to introduce, the structures still provide useful information by revealing the residues that shape the substrate- and metal-binding sites. In the crystal structures, PirXI appeared as a homotetramer with each monomer (49.5 kDa, $437 \mathrm{aa)}$ possessing an active site in which two divalent metal ions are bound. Soaking and cocrystallization studies showed that the ring-opened xylose binds in between two fully conserved tryptophan residues (Trp50 and Trp189) which play a role in the correct positioning of the substrate for catalysis [22,30]. Of the two active site metals, one $\left(M_{1}\right)$ is responsible for substrate binding while the other $\left(M_{2}\right)$ is essential for catalysis by polarizing the $\mathrm{M}_{2}$-bound catalytic water that protonates $\mathrm{O} 1$ of the substrate and consequently generates a carbocation on $\mathrm{C} 1$ promoting the $\mathrm{C} 2$ to $\mathrm{C} 1$ hydride shift $[22,31]$. The catalytic metal $\mathrm{M}_{2}$ moves during the reaction from the $\mathrm{M}_{2}$ a to the $\mathrm{M}_{2} \mathrm{~b}$ position, which is also visible in structures with certain combination of ligands: $5 \mathrm{NH} 7$ (xylose and $\mathrm{Mg}^{2+}$ ), 5NHC (xylulose and $\mathrm{Co}^{2+}$ ), 5NHD (xylose and $\mathrm{Ni}^{2+}$ ) and 5NHE (xylose and $\mathrm{Cd}^{2+}$ ) (Fig. 1) [22].

To examine if structure-inspired mutagenesis can contribute to obtaining improved xylose isomerase variants and to investigate the possibility that such mutants may be useful to identify catalytic properties relevant for in vivo performance, we now designed focused mutant libraries with mutations surrounding the residues involved in metal and substrate binding. These were screened for enhanced growth after expression in yeast. Improved enzymes were indeed discovered and their biochemical properties were investigated, in comparison 


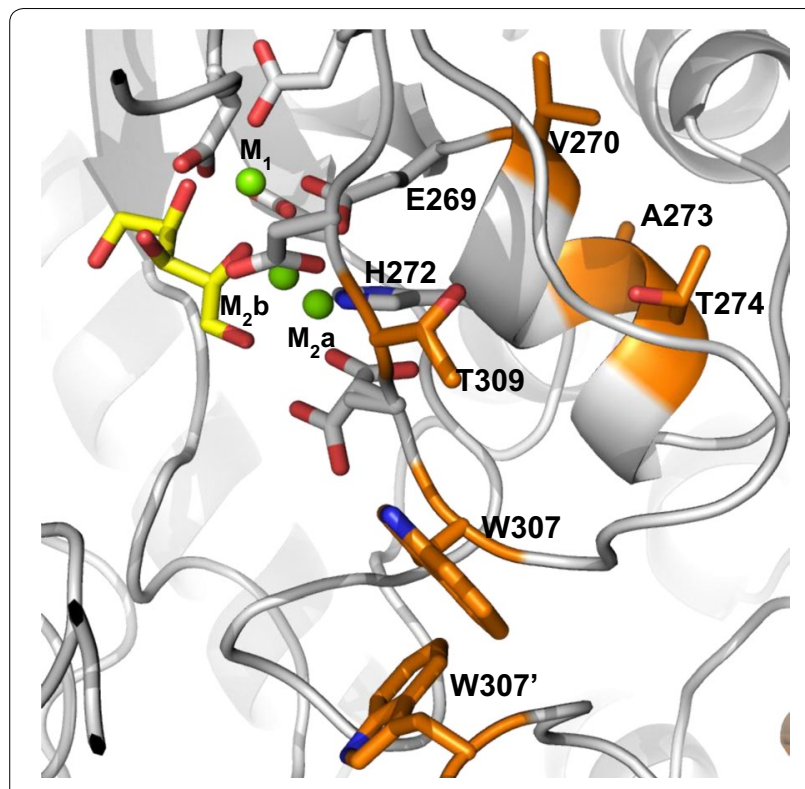

Fig. 1 Structure of the PirXI active site and design of mutant library LibM1. The figure shows the active site structure of PirXI with xylose (yellow) and $\mathrm{Mg}^{2+}$ ions (green spheres) bound (PDB: 5NH7). The target residues (orange) are located near the active site. The catalytic metal $\left(M_{2}\right)$ can occupy two positions $\left(M_{2} a\right.$ and $\left.M_{2} b\right)$. W307' is a residue from a neighboring subunit

to previously reported PirXI variants. The results indicate that small changes in catalytic properties may be accompanied by significant effects on xylose-supported growth. Furthermore, in vivo selection may govern mutations that improve xylose metabolism without changing kinetic properties measured under standard conditions with $\mathrm{Mg}^{2+}$ as the activating metal.

\section{Results}

\section{Library design}

To discover mutants of PirXI that enhance xylose metabolism, we designed and constructed small focused mutant libraries followed by in vivo screening for better variants. Recent structural and biochemical information was used to select target positions for mutagenesis, focusing on residues that surround the metal-binding sites. Replacing the second shell residues might have an effect on metal binding or reactivity and thereby influence the activity. The fully conserved metal-coordinating residues in PirXI are Glu233, Glu269, Asp297 and Asp340 for site $M_{1}$ and Glu269, His272, Asp308 and Asp310 for the catalytic metal $\mathrm{M}_{2}$. For the first library (LibM1), we targeted residues that are in close proximity of the $\mathrm{M}_{2}$ site. Five residues were selected: three (Val270, Ala273 and Thr274) that lie on the same helix as the metal binding residues (His272 and Glu269), and two (Trp307 and Thr309) that are on a nearby loop (Fig. 1). Amino acid diversity to be
Table 1 Design of PirXI library LibM1

\begin{tabular}{llll}
\hline Residue & Amino acids & Codons & ${\text { Freq. } \text { WT }^{\mathbf{a}}}^{\text {. }}$ \\
\hline V270 & VAG & GBT & 0.33 \\
A273 & A G & GST & 0.5 \\
T274 & TIKQ & AYC + MAA & 0.25 \\
W307 & WFYISTN & WHT+TGG & 0.14 \\
T309 & TSKRNQ & AVS + CAG & 0.29
\end{tabular}

${ }^{a}$ Codons at each position were selected in such a way that each desired amino acid, including the wild type, is included at a frequency of $1 /$ (total diversity)

introduced at each position was selected based on phylogenetic diversity, in silico-predicted stabilities of the mutants, and visual inspection of the predicted mutant structures. For phylogenetic input, a multiple sequence alignment was performed on 22 different class I and 100 class II XI sequences. Considering conservation scores and similarities between amino acid properties, the library diversity was decided. For example, residue Ala270 is fully conserved throughout all class II enzymes and therefore the diversity at this position was restricted to alanine and glycine to avoid extreme modifications. Changes in free energy of folding $\left(\Delta \Delta G_{\text {fold }}\right)$ of mutants relative to the wild-type enzyme were predicted using FoldX calculations [32]. Large decreases in predicted stability were used to dismiss mutations from the library design. The resulting LibM1 library included 1008 different variants (Table 1).

\section{Library construction}

To construct the LibM1 mutant library, at each target position a minimum number of partially undefined codons covering the selected set of amino acid substitutions was chosen using a spreadsheet implementing the CodonFinder routine [33]. The codons were selected in such a way that all the desired amino acids (including the wild-type residues) are incorporated at balanced coverage without introduction of undesired codons like stop codons. The 1008 LibM1 mutant library was covered by 8 partially undefined codons (Table 1).

Library DNA was obtained by generating gene fragments using PCR and subsequent cloning into $E$. coli-yeast shuttle expression vector (pRS426-URA) as described in "Methods". Transformation of the library DNA to E. coli resulted in over 8000 clones, of which pooled plasmid DNA was transformed to S. cerevisiae strain DS75543, producing 6000 colonies. Considering the library size of 1008 , these numbers are sufficient for near full library coverage [33]. Prior to yeast transformation, library diversity was confirmed by sequencing a mixture of plasmids isolated from the mixed collection of $E$. coli transformants. The sequencing results showed 
that all expected bases were incorporated at the correct positions, indicating sufficient library quality to proceed to screening.

\section{Screening for improved xylose utilization}

Library LibM1 was screened by growth competition of $S$. cerevisiae strain DS75543 transformed with library plasmids DNA. The entire collection of yeast transformants was inoculated into xylose medium and cells were cultivated with serial transfers to fresh xylose medium. Faster growing cells, which over time became dominant, were assumed to harbor an improved PirXI. Screening was performed both under aerobic and anaerobic (oxygenlimited) conditions, each in duplicate, as different variants can be expected depending on the metabolic status of the cells. For anaerobic growth, the cultures were kept oxygen-limited as described in "Methods". The effect of limited oxygen availability was reflected in the final cell densities $\left(\mathrm{OD}_{600}\right)$ of the cultures, which were $\sim 3$ and $>20$ for anaerobic and aerobic conditions, respectively. Anaerobic cultures initially required 8-9 days before growth occurred. A reduced lag time and/or increased growth rate was observed after multiple transfers with all four selection cultures, also in comparison to a control culture harboring only wild-type PirXI.

Both aerobic and anaerobic cultures were harvested after the 10th transfer and plasmids were isolated to evaluate the selected PirXI genes. Sequencing showed that all four cultures, i.e., both the aerobic duplicates and anaerobic duplicates, contained only one PirXI variant, which carried the mutations V270A and A273G.

\section{Effect of V270A-A273G PirXI on xylose utilization}

The consistent selection of the V270A-A273G variant from library LibM1 suggested that the screening method was reliable and sensitive. Nevertheless, it is possible that other events such as genomic mutations or variations in expression level were responsible for the improved growth of yeast carrying the V270A-A273G mutant PirXI. The replicon of the pRS416 vector used in this work is derived from yeast plasmid $2 \mu$, and plasmid copy numbers can vary from culture to culture [34]. Furthermore, in laboratory evolution of $S$. cerevisiae for growth on xylose, cells may acquire diverse chromosomal mutations that cause improved growth [35, 36]. To prove that in our case the selected mutations in the PirXI structural gene caused improved growth on xylose, the mutations were reconstructed by site-directed mutagenesis in the original PirXI gene and cloned in a vector that was not subjected to previous selection. S. cerevisiae DS75543 cells were then transformed with the freshly prepared constructs and their growth performance in a 96-well plate was monitored. We have repeated this process several times and consistently observed that the cells containing the mutant PirXI grow on xylose better compared to those containing wild-type PirXI (Fig. 2a). We have also observed that general growth performance of the cells slightly differs between experiments as well as between clones picked from a single transformation experiment. Figure $2 \mathrm{~b}$ shows a high variability in growth between 30 independent transformants despite their identical genotype. Nevertheless, the results clearly indicate that the V270A-A273G mutant PirXI improves

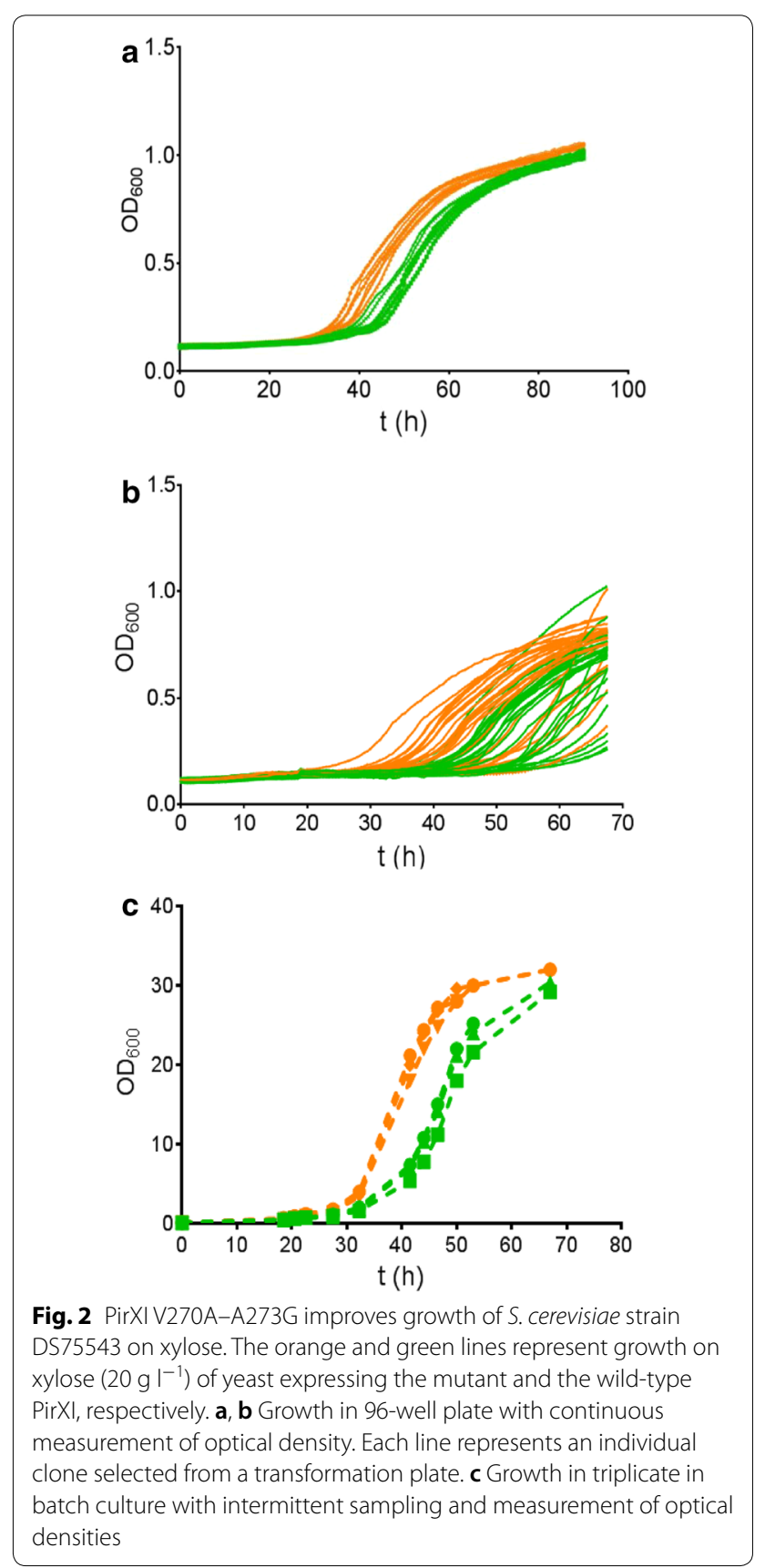


growth on xylose as compared to wild type, especially in the earlier phases of growth (Fig. 2b). On average, the mutant cultures started to grow earlier and more quickly reached their final density.

In 96-well plates, oxygen availability may not be well controlled and results could be influenced by evaporation. Therefore, a comparison between the wild-type and V270A-A273G XI variants was also performed using shake flask cultures with replicates inoculated with precultures from independent transformants. The resulting growth curves (Fig. 2c) confirm that the mutated PirXI is beneficial for growth on xylose. The specific growth rates $(\mu)$ were calculated from the exponential part of the curves, using the following equation for fitting: $\ln X=\ln X_{0}+\mu\left(t-t_{0}\right)$, where $X$ is the measured $\mathrm{OD}_{600}$ and $\mu$ is the rate. The average growth rates of the wild type and the mutant are $0.13 \pm 0.01$ and $0.18 \pm 0.01 \mathrm{~h}^{-1}$, respectively. These results show that the observed improved growth is due to the V270A-A273G mutations in PirXI, not by unidentified mutations elsewhere on the plasmid or in the chromosome of the selected transformants.

Yeast cells selected for good growth on xylose show high overexpression of PirXI [12, 15], which may be a metabolic burden for the cells and trigger selection of mutants with a higher activity:expression ratio in competition experiments. To examine if the PirXI mutations affected enzyme expression, we studied XI levels in cells grown on xylose. The specific activity with $100 \mathrm{mM}$ xylose measured with the cell-free extracts were $0.94 \mathrm{U} /$ $\mathrm{mg}$ and $0.54 \mathrm{U} / \mathrm{mg}$ for the wild type and the mutant, respectively. SDS-PAGE gels revealed that the expression levels for the wild-type and the mutant enzyme were similar (Fig. 3).

The uncertainty of in vivo metal binding properties of PirXI and metal content of the yeast cytoplasm makes it difficult to define a metal composition for assays that gives results reporting on in vivo performance. When we measured the PirXI activity of extracts of S. cerevisiae DS75543 cells without metal addition, the results indicated that the activity of the wild-type enzyme was almost twofold higher compared to the mutant. This unexpected observation could be caused by changes in PirXI metal composition during enzyme preparation and dilution, e.g., due to binding of metals released from organelles such as vacuoles or from changes in metalprotein interactions.

To examine if the individual mutations in the PirXI variant are both necessary for improved growth, we constructed the single mutants V270A and A273G and examined the effect on growth on xylose, particularly on the early growth phase. The growth curves indicate that the V270A mutation has a larger effect, showing much

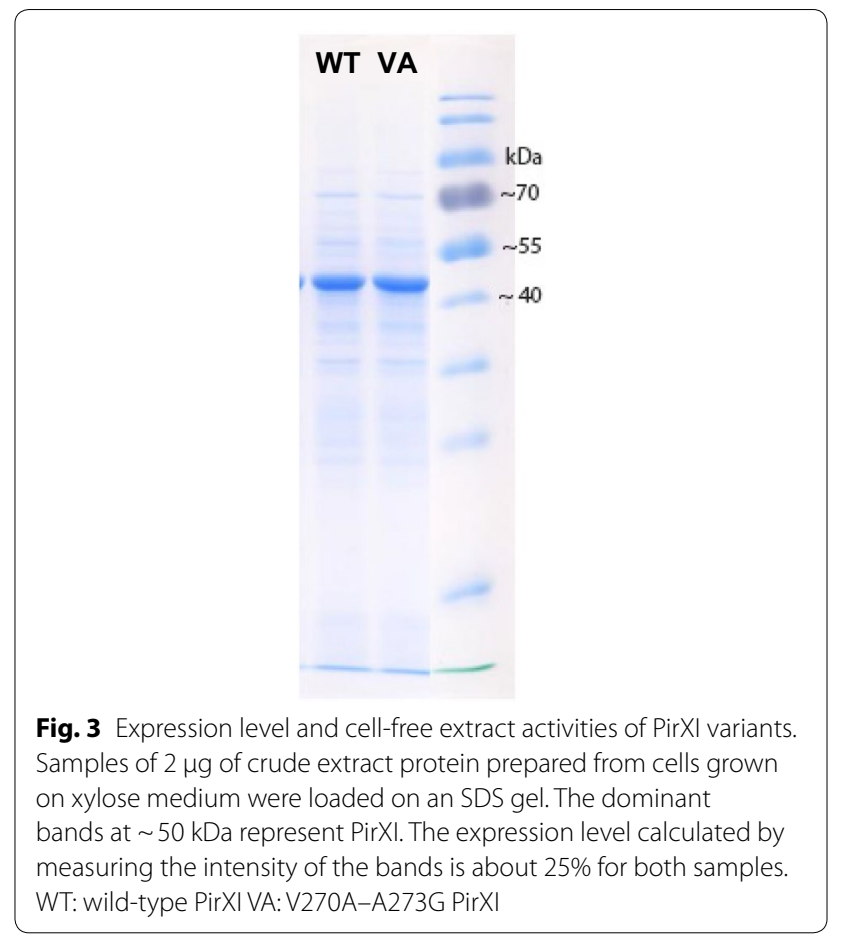

earlier initiation of exponential growth (Fig. 4), but also cells containing the A273G mutant PirXI showed a slight growth improvement compared to the cells expressing the wild-type enzyme.

\section{Kinetic properties of PirXI V270A-A273G}

We expected that the positive effect of the V270AA273G mutations on xylose-supported growth would be due to improved catalytic parameters, i.e., increased catalytic rate $\left(k_{\text {cat }}\right)$ or a better substrate affinity (reduced

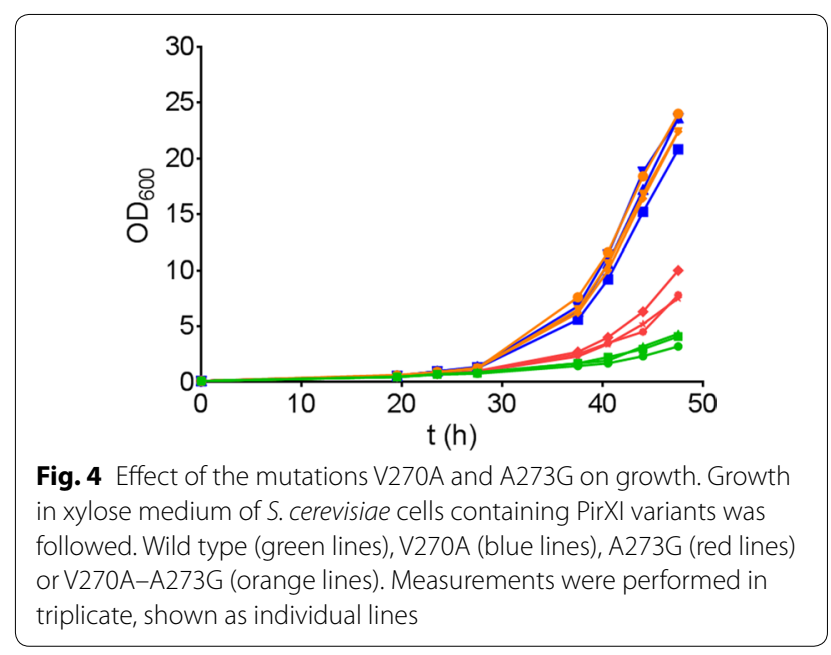

$\left.K_{\mathrm{M}}\right)$. Since PirXI can be activated by different divalent metals and activities depend on the type of metal that 
is bound [22], we measured XI activities with metals that the enzyme potentially binds in vivo as previously found by metal analysis $\left(\mathrm{Mg}^{2+}, \mathrm{Mn}^{2+}\right.$ or $\left.\mathrm{Ca}^{2+}\right)$ [21]. With none of these metals, the in vitro activities revealed an increased $k_{\text {cat }}$ or decreased $K_{\mathrm{M}}$ for the mutant enzyme in comparison to wild type. In contrast, the wild type performed better in the presence of all metals tested, showing slightly higher catalytic rates and substrate affinities (Table 2). Especially, with $\mathrm{Mn}^{2+}$, the activity of the mutant decreased 50\% compared to the wild type. This result indicates that an increase in specific activity with these metals, at least individually, is not responsible for the improved growth on xylose of yeast expressing V270A-A273G PirXI.

Besides the metal-dependence of the isomerase, metal affinities were considered as a possible cause of improved in vivo enzyme performance. We estimated metal affinities of the wild-type and the mutant enzyme by measuring the activation constant $\left(K_{\text {act }}\right)$ for each metal. This constant represents the metal concentration giving half-maximal enzyme activity. Since xylose isomerase requires two metals for activity, the value depends on the binding sites with the lowest affinity if both sites must be occupied. For measuring $K_{\text {act }}$ with $\mathrm{Mg}^{2+}$ and $\mathrm{Mn}^{2+}, 100 \mathrm{mM}$ xylose was used as substrate. In case of $\mathrm{Ca}^{2+}, 400 \mathrm{mM}$ xylose was used since the $K_{\mathrm{M} \text {,xylose }}$ of the PirXI-Ca ${ }^{2+}$ is very high (Table 2). The data showed that the V270A-A273G mutant showed slightly higher affinity for $\mathrm{Ca}^{2+}$ and $\mathrm{Mn}^{2+}$, whereas the wild-type enzyme has slightly higher affinity for $\mathrm{Mg}^{2+}$ (Table 2 ). However, the differences are small and do not indicate a shift in metal affinity as the cause of improved growth.

Metal affinity was also examined by measuring the effect of metal addition on PirXI thermostability since metal binding can stabilize metalloenzymes [37]. Effects on apparent melting temperatures were measured in the presence of different concentrations of metals using thermal shift assays (Fig. 5). The results showed that the apo forms of wild-type and V270A-A273G PirXI have a similar thermostability. Interestingly, whereas $T_{\text {m,app }}$ of the wild-type PirXI increased with metal concentration according to a hyperbolic saturation-like curve, the $T_{\text {m,app }}$ of the mutant enzyme was constant up to ca. $200 \mu \mathrm{M}$ of $\mathrm{Mn}^{2+}$ or $\mathrm{Ca}^{2+}$ added, with an increase at higher metal concentrations (Fig. 5a, c). In contrast, when $\mathrm{Mg}^{2+}$ was added, the thermostability of the mutant enzyme was not increased even at concentrations that were saturating for enzyme activity (Fig. 5b). The difference between the metal-concentration dependence of mid points of thermal shift assays and $K_{\text {act }}$ values measured in the presence of substrate suggests that substrate influences metal binding, as also observed when examining X-ray structures of the enzyme with different combinations of ligands [22]. Only in the presence of the substrate xylose

Table 2 Kinetic parameters of PirXI variants

\begin{tabular}{|c|c|c|c|c|c|}
\hline \multirow[t]{2}{*}{ PirXI variants } & \multirow[t]{2}{*}{ Metal } & \multirow[t]{2}{*}{$K_{\text {act }}(\mu \mathrm{M})^{c}$} & \multicolumn{3}{|c|}{ Kinetic parameters $^{a}$} \\
\hline & & & $k_{\text {cat }}\left(\mathrm{s}^{-1}\right)$ & $K_{\mathrm{M}}(\mathrm{mM})$ & $k_{\text {cat }} / K_{\mathrm{M}}\left(\mathrm{s}^{-1} \mathrm{M}^{-1}\right)$ \\
\hline \multirow[t]{3}{*}{ WT } & $\mathrm{Mg}^{2+}$ & $130 \pm 7$ & $2.2 \pm 0.1$ & $7.1 \pm 0.5$ & 310 \\
\hline & $\mathrm{Mn}^{2+}$ & $0.44 \pm 0.05$ & $6.9 \pm 0.2$ & $6.2 \pm 0.7$ & 1110 \\
\hline & $\mathrm{Ca}^{2+}$ & $230 \pm 20$ & $0.8 \pm 0.1$ & $1360 \pm 160$ & $0.4^{b}$ \\
\hline \multirow[t]{3}{*}{ V270A-A273G } & $\mathrm{Mg}^{2+}$ & $160 \pm 14$ & $1.8 \pm 0.1$ & $11.6 \pm 1.6$ & 155 \\
\hline & $\mathrm{Mn}^{2+}$ & $0.39 \pm 0.05$ & $2.8 \pm 0.1$ & $9 \pm 1$ & 310 \\
\hline & $\mathrm{Ca}^{2+}$ & $140 \pm 12$ & $0.28 \pm 0.01$ & $1760 \pm 120$ & $0.18^{b}$ \\
\hline \multirow{3}{*}{$\begin{array}{l}\text { S141N-T142S-A143S- } \\
\text { G174A }\end{array}$} & $\mathrm{Mg}^{2+}$ & $126 \pm 10$ & $1.6 \pm 0.2$ & $27 \pm 9$ & 60 \\
\hline & $\mathrm{Mn}^{2+}$ & $2.4 \pm 0.5$ & $4.9 \pm 1$ & $37 \pm 1.5$ & 132 \\
\hline & $\mathrm{Ca}^{2+}$ & $96.5 \pm 3.5$ & $0.06 \pm 0.01$ & $1200 \pm 300$ & $0.03^{b}$ \\
\hline \multirow[t]{3}{*}{ E15D-T142S } & $\mathrm{Mg}^{2+}$ & $190 \pm 10$ & $1.9 \pm 0.1$ & $22 \pm 2$ & 86 \\
\hline & $\mathrm{Mn}^{2+}$ & $2.1 \pm 0.3$ & $5 \pm 0.2$ & $53 \pm 4$ & 94 \\
\hline & $\mathrm{Ca}^{2+}$ & $50 \pm 10$ & $>0.02$ & N.A. & $0.018^{b}$ \\
\hline \multirow[t]{3}{*}{ N338C } & $\mathrm{Mg}^{2+}$ & $112 \pm 7$ & $4.2 \pm 0.2$ & $22 \pm 2$ & 191 \\
\hline & $\mathrm{Mn}^{2+}$ & $1.3 \pm 0.3$ & $7.2 \pm 0.2$ & $29 \pm 1.5$ & 248 \\
\hline & $\mathrm{Ca}^{2+}$ & $163 \pm 6.5$ & $0.78 \pm 0.2$ & $2800 \pm 800$ & $0.2^{b}$ \\
\hline
\end{tabular}

\footnotetext{
a All assays were performed at $30^{\circ} \mathrm{C}$ in $20 \mathrm{mM} \mathrm{MOPS}$ buffer, $\mathrm{pH}$ 7. Values are averages from three (wild type, V270A-A273G PirXI) or two (S141N-T142S-A143SG174A, E15D-T142S and N338C PirXI) independent measurements. The margins represent standard deviations

${ }^{b}$ Catalytic efficiencies obtained from slopes of Michaelis-Menten plots at $[S] \ll K_{M}$ because of the high $K_{M}$ for xylose in the presence of Ca ${ }^{2+}$ as activating metal

c The $K_{\text {act }}$ value is defined as the metal concentration giving half-maximal activity, as measured with a saturating concentration of $x y l o s e ~\left(100\right.$ mM in case of Mg ${ }^{+}$and $\mathrm{Mn}^{2+}, 400 \mathrm{mM}$ in case of $\mathrm{Ca}^{2+}$ )
} 

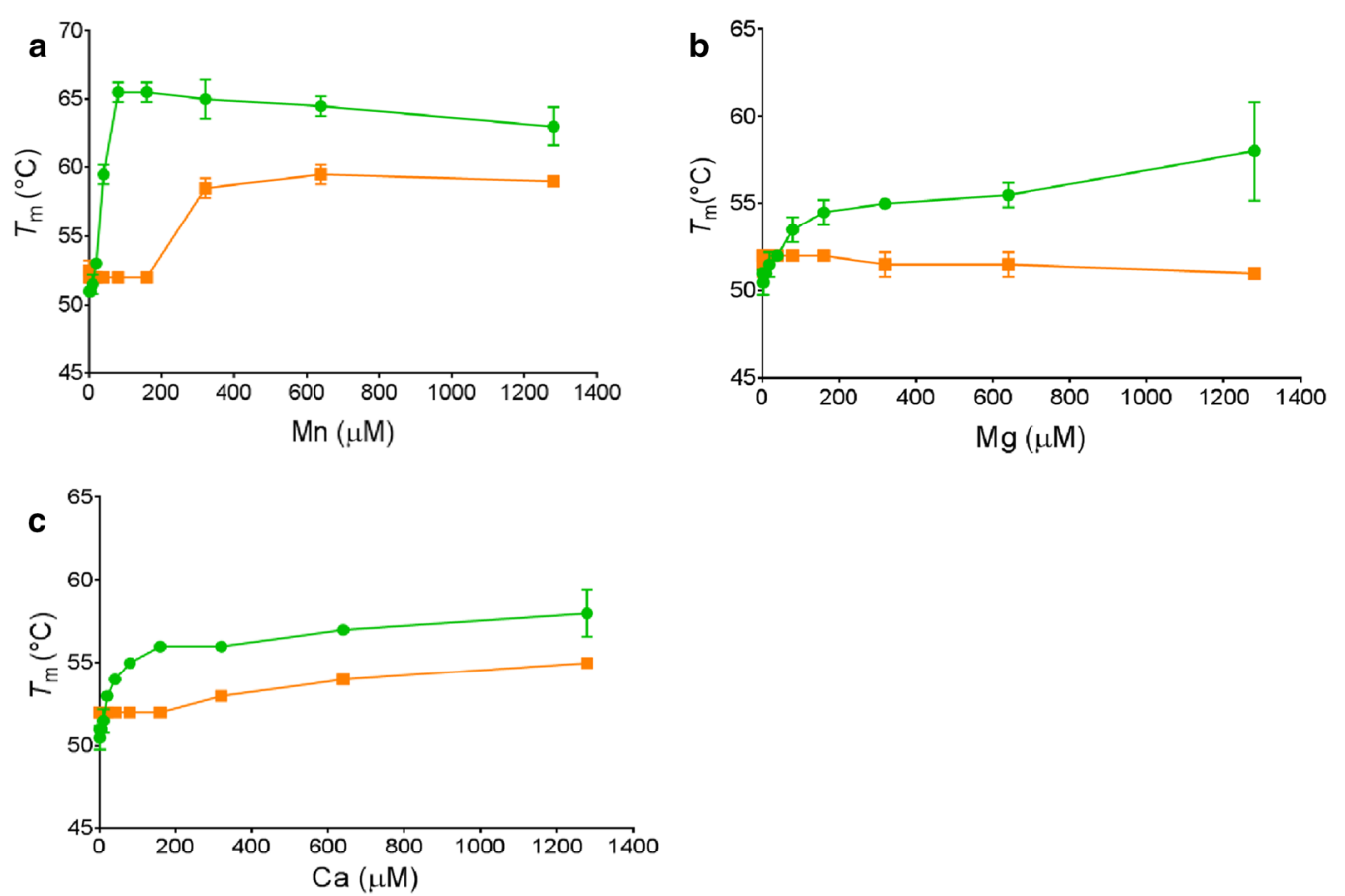

Fig. 5 Effect of metals on thermostability of PirXI. The graphs show the apparent melting temperatures of purified and EDTA-treated wild-type PirXI (green lines) and the V270A-A273G mutant PirXI (orange lines) in the presence of various concentrations of metals $\left(\mathbf{a} \mathrm{Mn}^{2+} ; \mathbf{b ~ M g}{ }^{2+} ;\right.$ and $\left._{\mathbf{c}} \mathrm{Ca}^{2+}\right)$. The $T_{m}$ values are averages from two independent measurements. $[E]=20 \mu \mathrm{M}$. Error bars represent standard deviations

both metal-binding sites in the crystal structures were occupied, whereas in xylitol- or glycerol-bound enzyme only the $\mathrm{M}_{1}$ metal site was occupied with a metal ion.

The metal content of yeast cells is complex and consists of both free metal ions and metal ions bound to macromolecules [38]. In general, in vivo metal binding by metalloproteins is controlled by mechanisms such as intracellular metal homeostasis, localization of protein folding, and activities of metal transporters and metallochaperones [39]. In a previous study, we showed that changes in intracellular metal composition affect metal composition of PirXI, which in turn influences catalytic performance [21]. PirXI isolated from yeast grown on xylose is mostly bound with $\mathrm{Ca}^{2+}$, which barely activates the enzyme. Therefore, a large portion of PirXI does not contribute to in vivo conversion of xylose. In contrast, the smaller fraction of PirXI that is bound with the strongly activating $\mathrm{Mn}^{2+}$ contributes most to the in vivo enzyme activity [21].

In view of the complex metal composition of S. cerevisiae, we measured the activities of the wild type and the V270A-A273G mutant in the presence of varying concentrations of $\mathrm{Mn}^{2+}$ and a fixed high concentration (1 $\mathrm{mM})$ of $\mathrm{Ca}^{2+}$ (Fig. 6). As expected, both variants showed higher activity with increasing concentration of $\mathrm{Mn}^{2+}$. Interestingly, the degree to which $\mathrm{Mn}^{2+}$ influenced the activity was different between the wildtype and the mutant enzyme. At low concentrations of $\mathrm{Mn}^{2+}(10-100 \mu \mathrm{M})$ and in the presence of $1 \mathrm{mM}$ $\mathrm{Ca}^{2+}$ the mutant enzyme showed slightly higher activity. This indicates that the activation of the mutant enzyme by $\mathrm{Mn}^{2+}$ in the presence of a high concentration of $\mathrm{Ca}^{2+}$ is improved. Even though the activity of the mutant is lower in the presence of $\mathrm{Mn}^{2+}$ or $\mathrm{Ca}^{2+}$ alone, at certain low $\mathrm{Mn}^{2+} / \mathrm{Ca}^{2+}$ concentration ratios, the V270A-A273G mutant enzyme is better activated than the wild type. These results suggest that differences in in vivo metal activation may be responsible for the improved growth of yeast cells expressing the V270A-A273G mutant PirXI.

\section{Crystal structures of PirXI wild type and V270A-A273G}

To examine possible structural changes in the V270AA273G PirXI, we solved and compared crystal structures of this variant and the wild-type enzyme purified from yeast (Fig. 7). The overall structures of the wild type and mutant enzyme are very similar and confirmed the mutations. In the mutant structure, the side chain of Phe 280 moves $\sim 0.5 \AA$ in the direction of Ala270. Due to the decrease in hydrophobicity and size the surrounding waters also shift towards Ala270. Mutation A273G shows no effect on the structure. There is no significant 


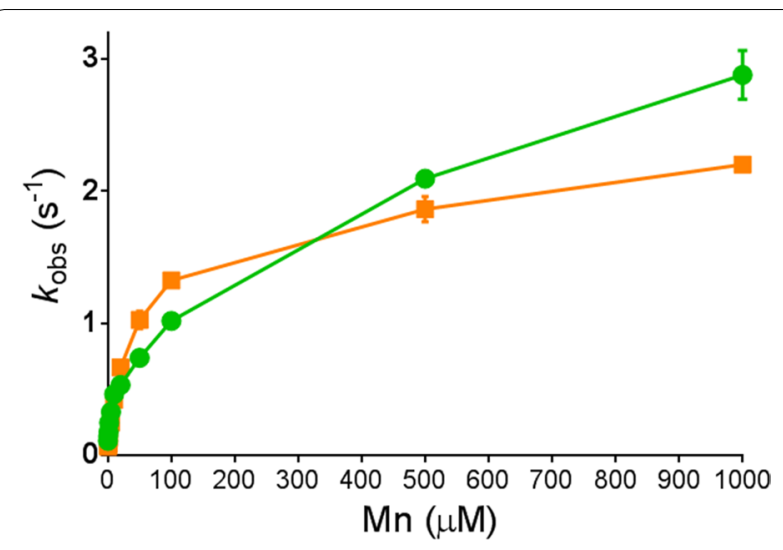

Fig. $6 \mathrm{PirXl}$ activity in the presence of $\mathrm{Ca}^{2+}$ and $\mathrm{Mn}^{2+}$. The activities of wild-type enzyme (green line) and the variant V270A-A273G (orange line) on $100 \mathrm{mM}$ xylose were measured in the presence of a mixture of $1 \mathrm{mM} \mathrm{Ca}^{2+}$ and various concentrations of $\mathrm{Mn}^{2+}$. The data represent the average values from duplicate measurements and the error bars represent standard deviations

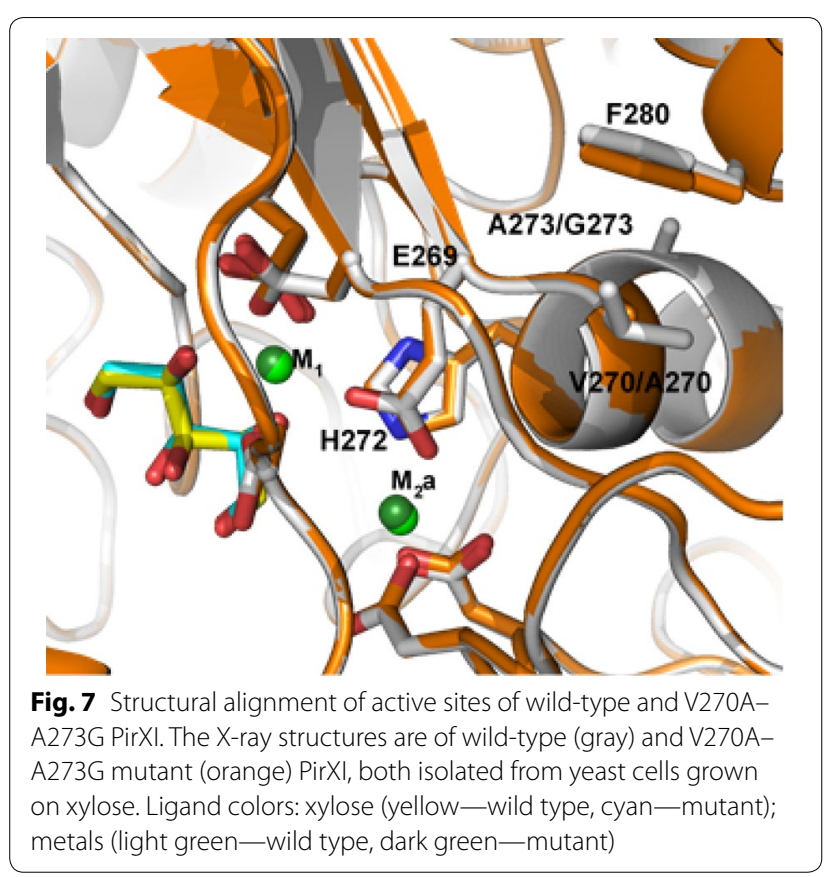

difference between the two structures to explain the improved in vivo performance of the mutant.

The enzyme crystals were prepared without removal or addition of metal ions so that only intrinsic metal ions are present. When the metals ions were refined as $\mathrm{Mg}^{2+}$, the Fo-Fc map showed unaccounted electron density at the metal positions, suggesting the presence of heavier ions. In an anomalous electron density map a clear signal was observed at the $\mathrm{M}_{1}$ and $\mathrm{M}_{2}$ a positions with $\sigma$ levels of 4.8 and 3.5, respectively. $\mathrm{Mg}^{2+}$ ions do not have an anomalous signal at the in-house used wavelength of $1.54 \AA$ A. However, a comparison with anomalous maps of previously determined structures [22] shows similar peak heights in the $\mathrm{Ca}-x y l o s e$ structure of PirXI (PDB code $5 \mathrm{NH} 8$ ). Therefore, metal ions at the $\mathrm{M}_{1}$ and $\mathrm{M}_{2}$ a positions were refined as $\mathrm{Ca}^{2+}$ with $100 \%$ occupancy resulting in a flat Fo-Fc map in both the wild-type and the doublemutant structures. The temperature factors (B-factors) of the two $\mathrm{Ca}^{2+}$ ions are 11.9 and 16.4 for the wild type and 11.4 and 19.3 for the mutant, which are lower than those of the surrounding residues. The distances of the coordinating side chains to the $M_{1}$ ion in the wild type PirXI and the mutant enzyme isolated from yeast are similar to those in the wild-type $\mathrm{Ca}$-xylose structure reported earlier (5NH8). These results indicate that most of the metal-binding sites of PirXI isolated from yeast are occupied by poorly activating $\mathrm{Ca}^{2+}$ ions, both in the wild-type and in the PirXI V270A-A273G mutant enzyme. Other metal ions, such as $\mathrm{Mg}^{2+}, \mathrm{Fe}^{2+}, \mathrm{Mn}^{2+}$ or $\mathrm{Co}^{2+}$, may be bound with low occupancy.

\section{Construction and screening of library LibM2}

A second library design for discovery of better xylose isomerase mutants focused on mutations in a stretch of six residues flanking the substrate binding site. In this case, to avoid the risk of improved growth by chromosomal mutations, we compared growth properties of yeast clones transformed with the library DNA (Table 3 ). The growth of library colonies on solid medium containing xylose as sole carbon source was monitored by visual inspection. Plasmid DNA was isolated from suspected positive (larger) clones, retransformed to yeast and rescreened.

Library design again included selection of target positions and diversity to be introduced at each position (Fig. 8). The residues at the six target positions (Ser141, Thr142, Ala143, Asn144, Val145 and Gly147) at the C5 side of the substrate interact with the substrate either directly or indirectly. Therefore, it was expected that modifying these residues can improve substrate binding

Table 3 Library LibM2 variants and codons

\begin{tabular}{llll}
\hline Residue & Variants & Codons & Freq. WT \\
\hline S141 & SIVATGN & RBC+AAC & 0.14 \\
T142 & TS & ASC & 0.5 \\
A143 & ATGS & RSC & 0.25 \\
N144 & NLQT & CWG + AMC & 0.25 \\
V145 & VLIC & VTT+TGC & 0.25 \\
G147 & GATS & RSC & 0.25 \\
\hline
\end{tabular}


and the catalytic rate. Residue Thr142 is fully conserved throughout all known xylose isomerase sequences. In the structure it is connected to $\mathrm{O} 5$ of the substrate via a water molecule. To keep this interaction, we limited the diversity at this position to Thr and Ser. In a previous study, the PirXI T142S mutation was discovered to improve the growth of yeast on xylose [14]. We preserved Phe146 as it is fully conserved and it plays an important role in keeping the active site hydrophobic. Together with Trp189 and other hydrophobic aromatic residues (Trp50 and Phe61) this promotes the hydride shift by shielding the hydride from solvent [40-42]. The resulting library consists of 3584 variants (Table 3).

The library was constructed using the same strategy as for library LibM1. The initial E. coli transformation yielded ca. 8000 colonies and the diversity was confirmed by sequencing a plasmid mixture obtained from pooled transformants. The subsequent transformation to $S$. cerevisiae DS75543 also resulted in over 8000 colonies. For identification of clones showing improved xylose utilization, transformed cells were washed from glucose plates and spread on xylose plates (see "Methods" for details). Many cells did not grow at all or started to grow very slow, causing visible differences between individual colonies, also for wild type. The latter indicated that other factors than PirXI activity influenced colony growth, for example the physiological status of transformed cells at the moment of plating. After three rounds of screening

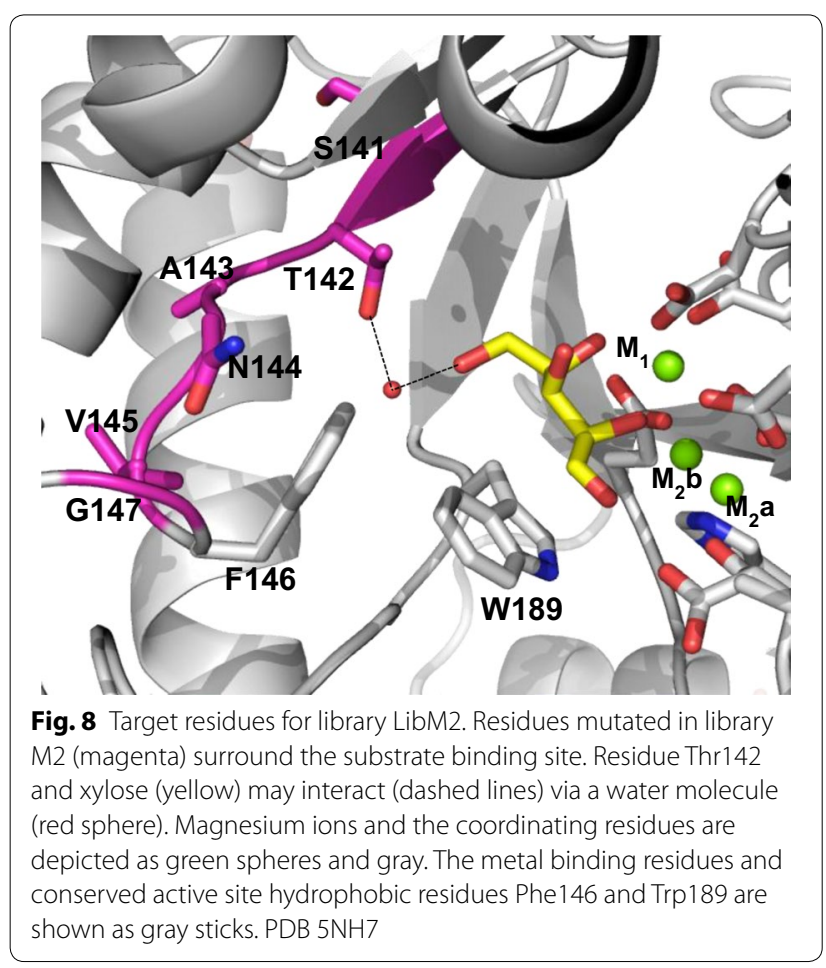

and retransformation, 46 colonies which showed superior growth were selected. To identify the best variant, growth in xylose containing liquid medium was measured using 96-well plates and compared to wild type. Most of the 46 variants reproducibly showed improved growth. The xylose isomerase genes from the 24 best growing variants were sequenced, revealing 10 different variants, one of which was wild type. The sequences that appeared most frequent (4-8 times) were reconstructed in a clean background and the effects on growth on xylose were evaluated after transformation to fresh DS75543 cells. Among these mutants, variant S1 (S141N-T142S-A143SG147A) consistently showed the biggest improvement of growth on xylose when several independent cultures were tested. As shown with variant V270A-A273G, the most significant effect of mutant S1 also appears to be on the earlier start of the growth while showing a slightly increased exponential growth rate (Fig. 9).

\section{Kinetic properties of PirXI S1}

The reconstructed PirXI mutant S1 was purified from E. coli and its activity was measured after reconstitution with different metals. As with variant V270A-A273G, the Michaelis-Menten kinetic parameters measured in the presence of $\mathrm{Mg}^{2+}, \mathrm{Mn}^{2+}$ or $\mathrm{Ca}^{2+}$ revealed reduced $k_{\text {cat }}$ values as compared to wild type (Table 2). The $K_{\mathrm{M}}$ for xylose was also several fold higher in the presence of $\mathrm{Mg}^{2+}$ or $\mathrm{Mn}^{2+}$ compared to the wild type. Furthermore, the $K_{\text {act }}$ values indicate that a shift in metal affinity does not promote better in vivo performance of the mutant enzyme as the affinity towards the most activating metal $\mathrm{Mn}^{2+}$ decreased, while the affinity towards $\mathrm{Ca}^{2+}$, which poorly activates the enzyme, increased.

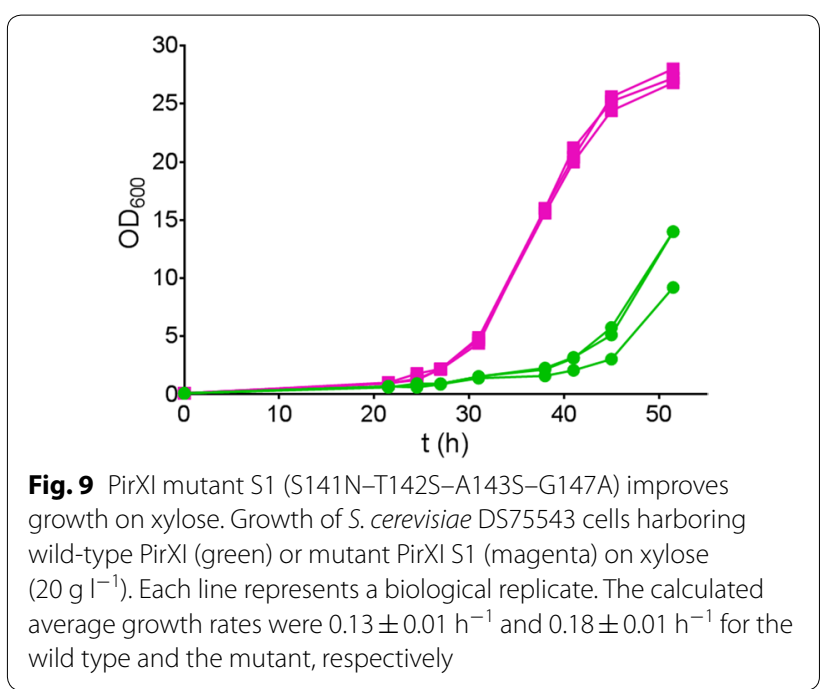




\section{Performance of other xylose isomerase mutants}

The results described above indicate that both libraries yielded PirXI mutants that caused accelerated growth on xylose. However, their properties exhibited disconnection between in vivo performance and in vitro catalytic properties. We further explored the ambiguous relation between in vivo and in vitro enzyme properties by studying PirXI mutants discovered independently in previous studies, using different $S$. cerevisiae host strains $[9,14]$. Using directed evolution with random mutant libraries, Lee et al. discovered PirXI variant E15D-T142S which increased the growth rate on xylose from $0.01 \mathrm{~h}^{-1}$ up to $0.06 \mathrm{~h}^{-1}$ [14]. Activities of these enzymes have only been measured with cell lysates, making a comparison difficult, but $K_{\mathrm{M}}$ values appear high. Later, Katahira et al. discovered that mutations at position N338, especially substitution N338C, improved growth of yeast on xylose. This mutation was effective not only in PirXI but also in related XIs [9]. It was reported that yeast cells carrying the N338C variant of PirXI consumed xylose 3-4 times faster than cells carrying wild-type PirXI. The catalytic properties of the mutant enzymes from these studies have not been described. Very recently, when our study was nearing completion, Seike et al. described mutations in XI from Lachnoclostridium phytofermentans (LpXI) that enhanced D-xylose metabolism [11]. The most effective mutations were T63I and V162A. The corresponding positions in PirXI are distant from the active site and the mutations were not examined here.

We constructed the two earlier PirXI mutants $[9,14]$, E15D-T142S and N338C, and confirmed that the variants are beneficial for PirXI-mediated growth of strain DS75543 on D-xylose as well (Fig. 10). Subsequently, we expressed the mutants in E. coli, purified the enzymes and measured kinetic parameters. For this, activities were determined with $\mathrm{Mg}^{2+}, \mathrm{Mn}^{2+}$ or $\mathrm{Ca}^{2+}$ added to the apoenzyme and the activation constants were also determined (Table 2). As with the new mutants described in the current paper, Michaelis-Menten parameters and metal affinities did not reflect the positive effect of the mutations on growth, with the exception of an increased $k_{\text {cat }}$ of the N338C mutant in the presence of $\mathrm{Mg}^{2+}$ and $\mathrm{Mn}^{2+}$. However, this enzyme also has a higher $K_{\mathrm{M}}$. This result shows that the disconnection between in vivo performance and in vitro properties of PirXI is not dependent on the screening strain or selection conditions.

\section{Discussion}

Improving D-xylose metabolism is an ongoing challenge for optimizing second-generation bioethanol production by engineered strains of $S$. cerevisiae. Important improvements in yeast performance have been achieved by evolutionary and metabolic engineering, which can

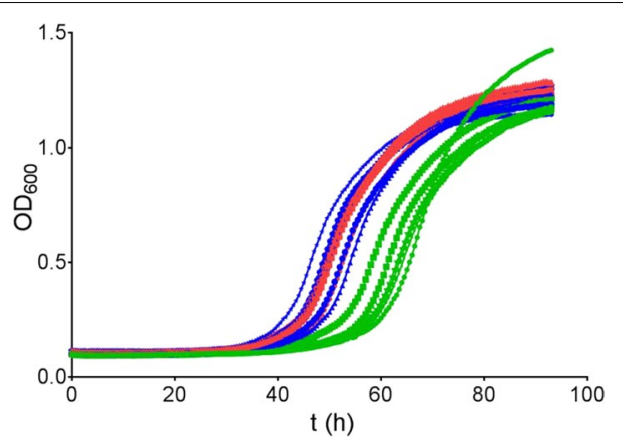

Fig. 10 Growth of S. cerevisiae expressing PirXI variants E15D-T142S or N338C. Growth of cells expressing the wild-type PirXI (green lines), a mutant PirXI E15D-T142S (red lines) or N338C (blue lines) on xylose $\left(20 \mathrm{~g} \mathrm{I}^{-1}\right)$ medium were measured using a microtiter plate reader. Individual lines represent biological replicates

influence different steps in central metabolism [16, 43], and by enhancing the xylose uptake system [44, 45]. Introduction of efficient enzymes for initial isomerization of D-xylose to D-xylulose is an equally important target. The incorporation of Piromyces xylose isomerase in xylulose fermenting yeast strains allowed xylose utilization $[12,13]$, but very high expression levels are needed for optimal performance, as indicated by gene amplification up to over 10 copies during adaptation [15, 21], leading to production of xylose isomerase at up to $25 \%$ of the cellular protein (Fig. 3). This suggested that the enzyme has poor in vivo kinetics and stimulated research aimed at discovering better xylose isomerase variants $[9,14]$.

In view of the catalytic properties of PirXI, the need for such a high expression level is unexpected. At the observed xylose isomerase content of $25 \%$, one would expect the isomerization reaction not to be growth-limiting. The relation between growth rate $\mu$ and xylose consumption $V$ can be expressed as:

$$
\begin{aligned}
& \mu=V \cdot Y, \text { with } \\
& V=[E] \cdot \frac{V_{\max } \cdot[S]}{K_{\mathrm{M}}+[S]},
\end{aligned}
$$

where $[E]$ is the enzyme content (ca. $0.1 \mathrm{~g}$ PirXI per $\mathrm{g}$ biomass estimated based on on $25 \%$ of the total protein being XI, and a total protein content of $0.4 \mathrm{~g}$ per $\mathrm{g}$ biomass [46, 47]), $V_{\max }\left(8.3 \mathrm{U} / \mathrm{mg}\right.$, from $k_{\text {cat }}=6.9 \mathrm{~s}^{-1}$ with $\left.\mathrm{Mn}^{2+}\right)$ and $K_{\mathrm{M}}(6.2 \mathrm{mM})$ have their usual meaning. $Y$ represents the yield on xylose $(0.25 \mathrm{~g}$ cell dry weight $/ \mathrm{g}$ xylose converted) estimated from a previous study performed with $S$. cerevisiae grown in a similar condition [48]. This predicts a PirXI activity at $[S]=1-10 \mathrm{mM}$ (reasonable intracellular substrate concentration) [49, 50] of 1-4.6 g xylose converted per g biomass per $h$, 
allowing a growth rate of $\mu=0.25-1.15 \mathrm{~h}^{-1}$. The experimentally observed growth was around $0.13 \mathrm{~h}^{-1}$, suggesting that xylose isomerase should not be rate limiting if it were fully active. PirXI is also well expressed and folded in vivo, which may be troublesome with other XIs, as illustrated by the extreme case of the xylose isomerase from Actinoplanes missouriensis, which in vitro looks catalytically superior to PirXI but fails to function in $S$. cerevisiae $[6,51]$. In view of earlier work on the effect of metals on PirXI activity and the observation that mutations influencing manganese homeostasis can improve xylose metabolism, we initially suspected that the modest activity of the enzyme might be due to suboptimal metal loading and that xylose utilization could be improved by engineering PirXI variants carrying mutations surrounding the metal-binding sites.

The discovery and engineering of XI variants that improve xylose metabolism has been pursued by different groups $[9,11,52]$. However, the connection between in vitro kinetics of xylose isomerase and yeast growth on xylose remains rather unclear. Recently, Seike et al. [11] compared xylose isomerases from different organisms and found that an enzyme from $L$. phytofermentans ( $L p \mathrm{XI})$ and two mutants thereof gave the highest xylose consumption rate even though its activity measured in cell lysates were not better than those of cells expressing PirXI [7]. Mutants of $L p X I$ that gave better xylose consumption were found. A higher activity $\left(V_{\max }\right)$ and lower $K_{\mathrm{m}}$ were found with a double mutant of $L p \mathrm{XI}$, but the experiments were done with whole cell lysates reconstituted with $\mathrm{Mg}^{2+}$, so a comparison of intrinsic kinetic parameters is difficult. Similarly, an XI from Burkholderia cenocepacia which gave higher in vitro activity of cell lysates compared to PirXI and LpXI [52] did not seem beneficial for xylose fermentation [11]. In the same context, a recently discovered XI from the gut bacterium $R$. speratus was found to be better for xylose fermentation than PirXI, but this could not be explained by differences in in vitro catalytic performance [9].

We initially expected that selection of faster growing yeast strains from libraries expressing mutants of PirXI would give variants with improved kinetic parameters (higher $k_{\text {cat }}$, lower $K_{\mathrm{M}}$ ) with $\mathrm{Mn}^{2+}$. Also changes in metal binding affinity or shifts in metal preference could be expected. Two focused libraries with good diversity at the target positions were constructed and improved mutants were indeed obtained by batch culture selection and plate screening for higher growth rates. From the first library (LibM1), the same V270A-A273G PirXI variant was repeatedly retrieved, both from aerobic and anaerobic duplicate cultures. This indicates that the features of PirXI which limit growth of yeast on xylose are not dependent on oxygen availability, and that aerobic screening is possible to discover mutations that contribute to anaerobic xylose metabolism as well. Examining a reconstructed V270A-A273G PirXI mutant demonstrated that the improved performance was due to the mutations in the PirXI structural gene. A second focused library (LibM2) was screened on solid xylose medium and led to the discovery of the fourfold mutant S141N-T142S-A143S-G174A. Again, the contribution of chromosomal mutations was excluded. After discovery of these new mutants, we investigated the relation between enzyme kinetics and improved growth, an issue that is also still open for earlier mutants of PirXI which were obtained by enrichment after error-prone PCR [9, $14]$. With the reconstructed mutant genes expressed in a clean expression vector and host, we found that all four PirXI variants improved growth on xylose of $S$. cerevisiae DS75543, a strain different from the one used earlier by others $[9,14]$.

Using purified proteins of the two new mutants (V270A-A273G and S141N-142S-A143S-G174A) as well as of the earlier variants (E15D-T142S and N338C) [9, 14], we measured activities and kinetic parameters under a variety of conditions. Activities at physiological $\mathrm{pH}(\sim 7)$ and temperature $\left(30{ }^{\circ} \mathrm{C}\right)$ initially did not reveal any obvious features of the mutant enzymes that account for faster growth on xylose. Measurements with enzyme variants that were reconstituted with a single type of metal indicated that the mutants under these conditions had no advantage over the wild type, but likely this incompletely reflects in vivo conditions where the enzyme binds mixtures of metals and needs to function in the complex environment of the cytoplasm. Metal binding in the cytosol of $S$. cerevisiae is dependent on metal availability as well as binding affinities, which differ among xylose isomerases and between the two binding sites $[53,54]$. The most pronounced yet small effect was the increased $k_{\text {cat }}$ of the N338C mutant with $\mathrm{Mn}^{2+}$ and $\mathrm{Mg}^{2+}$ as activating metals, as well as a slightly improved activity of the V270A-A273G variant in the presence of a low concentration of $\mathrm{Mn}^{2+}$ and a high concentration of $\mathrm{Ca}^{2+}$. Thus, at certain concentrations of these two metals, the mutant enzyme can be more active than the wild type. With all PirXI variants examined, $\mathrm{Mn}^{2+}$ gave much better activity than $\mathrm{Ca}^{2+}$, emphasizing the importance of $\mathrm{Mn}^{2+}$ homeostasis for enzyme activity, which was also shown in our previous study where cellular manganese content was enhanced by mutations in a metal transporter [21].

We also found that the main metal in PirXI isolated from yeast is $\mathrm{Ca}^{2+}$ and only a small amount of $\mathrm{Mn}^{2+}$ is present [21]. This metal composition of the enzyme is far from optimal for catalysis as $\mathrm{Ca}^{2+}$-bound PirXI shows very high $K_{\mathrm{M}}$ for xylose, over 200-fold higher than with 
the catalytically preferred $\mathrm{Mn}^{2+}$ (Table 2). A shift in metal preference thus can explain improved in vivo performance, although establishing a quantitative correlation is impossible since in vivo metal binding of the enzyme is difficult to predict and measure, especially because the enzyme has two metal-binding sites with different affinities, with one site essential for catalysis yet probably only occupied when substrate is bound [22]. Indeed, the fact that the metal composition of PirXI does not strictly follow the apparent metal affinities $\left(K_{\text {act }}\right)$ and intracellular metal composition of $S$. cerevisiae indicates a complex in vivo metal binding mechanism of the enzyme [21]. Changes in metal binding were also suggested by a different response of the wild-type and mutant PirXIs to metal titration followed by thermal shift assays. While the thermostability of wild type increases with increasing metal concentrations, the mutant $\mathrm{V} 270 \mathrm{~A}-\mathrm{A} 273 \mathrm{G}$ required high concentrations of the metals $(>200 \mu \mathrm{M})$ for an effect on thermostability, illustrating that the typical increase in thermostability of a metalloenzyme upon metal binding is abolished by the mutations. It is possible that binding of metals is affected by the availability and binding of substrate $[22,40]$.

Even though the mutations changed the metal specificity of the enzyme, increasing the affinity for both $\mathrm{Mn}^{2+}$ and $\mathrm{Ca}^{2+}$ slightly, crystal structures of mutated PirXI isolated from yeast showed that the enzyme still has $\mathrm{Ca}^{2+}$ ions occupying both metal-binding sites. It is interesting that the structure of PirXI isolated from $E$. coli showed a metal composition quite different from that of the enzyme produced in yeast, with in case of the yeast enzyme only the $\mathrm{M}_{1}$ binding site being occupied by $\mathrm{Fe}^{2+}$ (25\%), $\mathrm{Ca}^{2+}(40 \%)$ and $\mathrm{Mg}^{2+}(35 \%)$ as estimated from structural refinement and with the $\mathrm{M}_{2}$ site left empty. These data confirm that in yeast most of the enzyme is loaded with the catalytically impractical $\mathrm{Ca}^{2+}$ ions, with a high impact on the in vivo performance of the enzyme. This indicates that conclusions about increased $V_{\max }$ values of evolved XIs should be considered with care, especially in case of measurements performed with cell-free extracts instead of purified enzyme and in the presence of an excess of added $\mathrm{Mg}^{2+}$, which is routinely used in XI assays $[11,14]$.

Other factors that might possibly influence in vivo enzyme performance include enzyme stability (lifetime), compartmentalization, and interaction with other cellular macromolecules. PirXI originates from a heterologous host causing the enzyme to be not evolutionarily optimized for functioning in S. cerevisiae. The distribution of metals over cellular compartments and the sequestration of metals by other macromolecules are likely to have a major impact on the metal availability for PirXI in vivo [55]. Such differences in enzyme properties beyond kinetic parameters may influence the performance of xylose isomerase variants. Differences between host strains, as well as variations in cultivation conditions and assay conditions make it difficult to compare the performance of xylose isomerases in yeast xylose metabolism.

\section{Conclusion}

As part of developing efficient second-generation bioethanol production, there have been efforts to engineer xylose isomerase variants that improve growth of S. cerevisiae on xylose. We found that design of focused libraries of Piromyces xylose isomerase, based on inspection of the crystal structure, followed by growth-based screening, gives mutants that improve the growth of yeast on xylose. The mutations differ from those found earlier in random mutant libraries constructed by errorprone PCR. The new mutants described here and the two mutants discovered earlier, did not show improved xylose isomerization kinetics when tested in vitro with in the presence of an excess of single metals. Yet, metal occupation of the enzyme is of key importance as indicated by the low in vivo activities and the high calcium content found by metal analysis and X-ray crystallography of xylose isomerase isolated from yeast. Small differences in relative metal affinities and activities can explain the improved growth caused by mutations in the second shell of metal coordination. Rational redesign of xylose isomerase for better in vivo performance would require a theoretical framework that describes xylose isomerase structure-activity relations as a function of metal incorporation and activation.

\section{Methods}

\section{Strain and plasmid construct}

The E. coli strain used in this work is NEB $10 \beta$ (New England Biolab). S. cerevisiae strain DS75543 is derived from RWB217 [16] and was constructed for xylose fermentation by genetic engineering and further improved for growth rate on xylose by laboratory evolution. Plasmids bearing the XKS1 and PirXI expression cassette were cured from the strain, and an XKS1 overexpression cassette was re-introduced by integration in the yeast genome. The relevant genotype of DS75543 is the following: MATa, ura3-52, leu2-112, gre3::loxP, loxPPtpi::TAL1, loxP-Ptpi::RKI1, loxP-Ptpi::TKL1, loxPPtpi::RPE1, TY1::Padh1XKS1+LEU2.

A yeast codon-optimized xylose isomerase geneexpression cassette, $\mathrm{P}_{T P I I} X y l A_{-} \mathrm{T}_{C Y C l}$, was obtained from Prof. J.T. Pronk, TU Delft, and cloned into the $2 \mu$ plasmid pRS426-URA using SacI and SalI restriction sites. For E. coli expression, a $\mathrm{pBAD} /$ myc-His-derived plasmid containing E. coli codon-optimized XylA was used as described in our previous study [22]. 


\section{Library construction}

The library construction strategy is schematically shown in Fig. 11. A mutant PirXI library was created by cloning XylA fragments containing mutations into the pRS426URA vector. The fragments and the vector were designed to contain $>20$ bp overlaps for use of the Gibson assembly method for cloning. Appropriate partially undefined codons for generating the library were designed using a spreadsheet-based site-restricted library design tool called CoFinder [33]. Using primers containing the partially undefined codons and pRS426_pTPI_XylA as the template, PCR was performed to generate the library fragments. For creating the backbone, linearized pRS426_pTPI_XylA was used as the PCR template. The plasmid was linearized by using a restriction enzyme that cuts the DNA once outside of the backbone region. An AatII site was created for library LibM1 and the single existing BglII site was used for library LibM2. For both the fragment and the backbone generation, Phusion high-fidelity polymerase (ThermoFisher) was used and the instructions of the manufacturer were followed for the PCR reactions. After PCR, template fragments were degraded by incubating the reaction mixtures with $1 \mu \mathrm{l}$ of $\mathrm{DpnI}$ at $37^{\circ} \mathrm{C}$ for $3 \mathrm{~h}$. For Gibson assembly of library fragments and backbone, a total of $~ 100$ ng DNA was added at a 1:3 ratio of purified backbone to gene fragments. The library DNA fragments were mixed proportionally to the number of variants that is theoretically carried by each fragment. The assembly reactions were performed following the protocol established by Gibson et al. [56].
Subsequently, three aliquots of $100 \mu \mathrm{l} E$. coli were transformed with $10 \mu \mathrm{l}$ reaction mixtures and transformants were selected on LB agar plates containing $50 \mu \mathrm{g} \mathrm{ml}^{-1}$ ampicillin. Plasmids from the entire $E$. coli transformant mixture were isolated, sequenced to confirm the diversity of the library and transformed to S. cerevisiae.

\section{In silico prediction of enzyme stability}

The relative folding free energy differences $\Delta \Delta G^{\text {Fold }}$ between PirXI wild type and variants were predicted using FoldX calculations [32] based on the X-ray structure of the wild-type enzyme (5NH7) and in silico-generated mutants.

\section{Site-directed mutagenesis}

Selected PirXI variants for expression in E. coli and S. cerevisiae were reconstructed by QuikChange site-directed mutagenesis. For PCR, PfuUltra II Hotstart master mix (Agilent) was used following the manufacturer's instructions. Subsequently, $1 \mu \mathrm{l} \mathrm{DpnI}$ was added to the reaction mixture, which was incubated at $37^{\circ} \mathrm{C}$ for $3 \mathrm{~h}$. Next, $10 \mu \mathrm{l}$ of the reaction mixture were used to transform $100 \mu \mathrm{l}$ NEB $10 \beta$ cells.

\section{Transformation}

For E. coli transformation $\mathrm{RhCl}_{2}$-competent $\mathrm{NEB} 10 \beta$ cells were used and a standard heat-shock protocol was applied. S. cerevisiae transformation was performed using the LiAc/SS carrier-DNA/PEG method. The protocol established by Gietz et al. was followed [57], adjusting

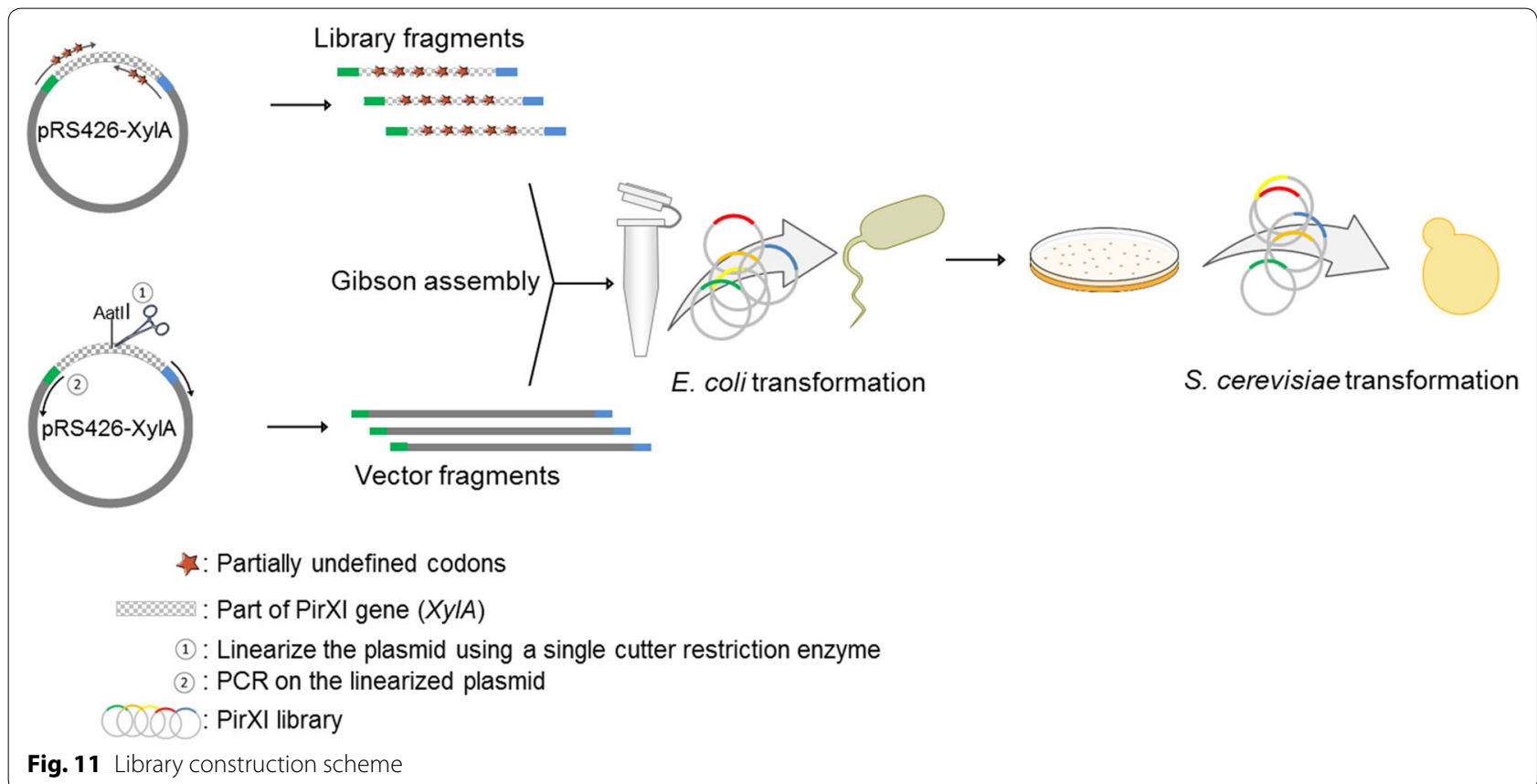


the duration of heat-shock to that works best for the DS75543 strain which was approximately $1 \mathrm{~h}$.

\section{Yeast cultivation}

For all yeast growth experiments a defined medium prepared according to Verdyun et al. [58] was used, either supplemented with $20 \mathrm{~g} \mathrm{l}^{-1}$ glucose (glucose medium) or $20 \mathrm{~g} \mathrm{l}^{-1}$ xylose (xylose medium) unless stated otherwise. For solid medium $2 \%$ agar was added prior to autoclaving. Cells were pre-grown on glucose medium and leftover glucose was washed away with $\mathrm{dd}_{2} \mathrm{O}$. The washed cells were resuspended in xylose medium and diluted appropriately. On-line growth measurements of S. cerevisiae were performed using a microplate reader (Synergy H1, BioTek). For this, $200 \mu \mathrm{l}$ xylose medium was used in 96-well cell culture plates (Eppendorf) and samples from pre-cultures were added to an $\mathrm{OD}_{600}$ of 0.02 . Plates were covered with an optical-clear gas-permeable seal (Breath-easy, Diversified Biotech). Cultures were grown at $30{ }^{\circ} \mathrm{C}$ with continuous shaking (linear, $731 \mathrm{pm}$, $2 \mathrm{~mm}$ shaking amplitude) and the $\mathrm{OD}_{600}$ was measured with 30-min intervals. For off-line growth measurements cells were cultivated at $30{ }^{\circ} \mathrm{C}$ in 100 -ml shake flasks containing $25 \mathrm{ml}$ xylose medium and the optical densities at $600 \mathrm{~nm}$ was followed in a spectrophotometer using plastic cuvettes.

\section{Library screening by competitive growth}

After transformation, yeast cells were plated on solid glucose medium. All transformants were collected by gently scraping the colonies from the plate and divided for duplicates of aerobic and anaerobic screening. Prior to the screening, cells were pre-grown on glucose until the mid-exponential phase. For the aerobic screening, precultures prepared were diluted in $50 \mathrm{ml}$ xylose medium to an $\mathrm{OD}_{600}$ of $\sim 0.1$ and grown at $30{ }^{\circ} \mathrm{C}$ with shaking at $135 \mathrm{rpm}$. For anaerobic growth, cells prepared from pre-cultures were inoculated in $100 \mathrm{ml}$ xylose medium supplemented with $420 \mathrm{mg} \mathrm{l}^{-1}$ Tween 80 and $10 \mathrm{mg} \mathrm{l}^{-1}$ ergosterol. To keep the conditions oxygen-limited, the cultures were grown in 100-ml Schott glass bottles which were kept air-tight with a rubber stopper and a glass airlock. Autoclaved medium was flushed with argon for at least $20 \mathrm{~min}$ prior to inoculation. The cultures were grown at $30{ }^{\circ} \mathrm{C}$ without shaking, but occasionally stirred briefly to keep homogenous cell suspensions. For aerobic and anaerobic screening, transfers to fresh xylose medium were carried out when the cultures reached an $\mathrm{OD}_{600}$ of 8-10 and 2, respectively. After the 10th transfer, cultures were harvested to investigate the evolved library diversity. Cells were pre-treated with zymolyase (Amsbio) and plasmids were isolated using a miniprep kit (Qiagen). The isolated plasmids were transformed to $E$. coli to produce sufficient plasmid DNA for sequencing. The plasmids were isolated from E. coli transformants using a miniprep kit and sequenced by GATC (Konstanz, Germany).

\section{Library screening by comparative growth}

All library transformants were collected from glucose medium plates, washed and diluted with sterile $d_{d d H_{2}} \mathrm{O}$ and plated on solid xylose medium. After 2-3 days of incubation, around 100 colonies that grew faster were selected by comparing colony sizes with cells expressing wild-type PirXI. Plasmids were isolated from selected transformants as described above and retransformed into fresh non-evolved yeast cells. The selection procedure was repeated twice and after the last round of yeast transformation and selection approximately 200 random colonies were picked and replicated both on glucose and xylose plates. Several colonies harboring wild-type PirXI were included as controls. Next, 46 colonies that grew faster than the controls on the xylose medium were selected and the corresponding colonies were picked from the glucose plate. The growth of the selected transformants in liquid xylose medium was measured in a 96-well plate using a microtiter plate reader. The growth experiments with these 46 transformants and wild-type PirXI carrying clones were performed in duplicate. The 24 best growing colonies were selected, plasmids were isolated and sequenced as described above.

\section{Enzyme expression in E. coli and purification}

For in vitro enzyme analysis, PirXI variants were expressed in E. coli and purified. NEB $10 \beta$ cells harboring PirXI variants were grown in TB medium (12 g/l tryptone, $24 \mathrm{~g} / \mathrm{l}$ yeast extract, $5 \mathrm{ml} / \mathrm{l}$ glycerol, $2.31 \mathrm{~g} / \mathrm{l}$ $\mathrm{KH}_{2} \mathrm{PO}_{4}$, and $16.43 \mathrm{~g} / \mathrm{l} \quad \mathrm{K}_{2} \mathrm{HPO}_{4} \cdot 3 \mathrm{H}_{2} \mathrm{O}$ ) containing $50 \mu \mathrm{g} \mathrm{ml}^{-1}$ ampicillin at $37{ }^{\circ} \mathrm{C}$. For inducing expression, $0.2 \%(\mathrm{w} / \mathrm{v}) \mathrm{L}$-arabinose was added and the cells were cultivated for $16 \mathrm{~h}$ at $37^{\circ} \mathrm{C}$. The cells were harvested by centrifugation and purification of overexpressed XI was done as described in previously [22].

\section{In vitro enzyme activity and metal affinity}

For measuring the XI activity in the presence of different metal cofactors, purified enzymes were first incubated overnight with $10 \mathrm{mM}$ EDTA. Subsequently, any metalEDTA complex and excess EDTA were removed by buffer exchange to $20 \mathrm{mM}$ MOPS (pH 7.0) using EconoPac 10-DG desalting columns (Bio-Rad). All enzyme activities were measured with sorbitol dehydrogenase $(\mathrm{SDH}$, Roche Diagnostics $\mathrm{GmbH}$ ) - coupled assay at $30{ }^{\circ} \mathrm{C}$ and $\mathrm{pH} 7.0$ (20 mM MOPS). The reactions were followed 
either using a spectrophotometer ( $1 \mathrm{ml}$ mixtures) (Jasco) or with a microplate reader in $(200 \mu$ reactions) (Synergy $\mathrm{H} 1$, BioTek). All reactions contained $0.15 \mathrm{mM} \mathrm{NADH}$, $1 \mathrm{mM}$ divalent metal, $1.5 \mathrm{unit} / \mathrm{ml} \mathrm{SDH}$ and D-xylose. The mixtures were incubated at $30{ }^{\circ} \mathrm{C}$ for $5 \mathrm{~min}$ and the reactions were initiated by addition of $0.05-0.2 \mu \mathrm{M}$ apo XI. For measuring the activation constant for each metal, activities of XI on $100 \mathrm{mM}$ (for $\mathrm{Mg}^{2+}$ and $\mathrm{Mn}^{2+}$ ) or $400 \mathrm{mM}$ (for $\mathrm{Ca}^{2+}$ ) D-xylose in the presence of various concentrations of metals were determined using a microplate reader. Various concentrations of metals and PirXI were used according to the level of metal-dependent activity of the enzyme.

\section{Thermostability measurement}

Thermostability of PirXI variants were determined by measuring an increase in fluorescence of Sypro Orange (Life Technologies, Carlsbad, CA, USA) during thermal unfolding [59]. The change of fluorescence emission at $575 \mathrm{~nm}$ was measured with CFX RT-PCR system (Biorad) while increasing the temperature from 20 to $90{ }^{\circ} \mathrm{C}$ at a rate of $0.5{ }^{\circ} \mathrm{C} \mathrm{min}{ }^{-1}$. In order to evaluate the metal affinity of PirXI variants, various concentrations of divalent metal ions $\left(\mathrm{MgCl}_{2}, \mathrm{MnCl}_{2}\right.$ or $\left.\mathrm{CaCl}_{2}\right)$ ranging from 0 to $1.28 \mathrm{mM}$ were included in $25-\mu \mathrm{l}$ reaction mixtures containing $1 \mathrm{mg} / \mathrm{ml}$ apo PirXI and $2 \times$ of Sypro Orange dye.

\section{Cell extract activity}

Saccharomyces cerevisiae cells grown on xylose were harvested, washed with sterile $\mathrm{ddH}_{2} \mathrm{O}$ and resuspended with lysis buffer containing $20 \mathrm{mM}$ MOPS (pH 7.0) and $100 \mathrm{U} / \mathrm{g}$ cells $(\mathrm{w} / \mathrm{w})$ zymolyase (Amsbio). The cell suspension was incubated at $30{ }^{\circ} \mathrm{C}$ with mild shaking at $50 \mathrm{rpm}$ for $20 \mathrm{~min}$. Next, the zymolyase-treated cells were disrupted by vortexing for $30 \mathrm{~s}$ with an equal volume of glass beads followed by cooling on ice for $1 \mathrm{~min}$. The process was repeated five times. The cell lysate was spun down by centrifugation at $17,000 \times g$ and the supernatant was collected as the cell-free extract (CFE). The total protein concentration of the CFE was measured by the Bradford assay using bovine serum albumin (BSA) as the standard. Activity of the CFE on $100 \mathrm{mM}$ xylose was measured by the SDH-coupled assay as described above without addition of any metals.

\section{Enzyme expression levels}

Crude extracts of $S$. cerevisiae expressing either wildtype or V270A-A273G PirXI grown on xylose were prepared by lysing the cells as described above. Total protein concentrations were determined by the Bradford assay and $2 \mu \mathrm{g}$ of protein from each cell extract was used for
SDS-PAGE analysis. Along with the crude extract samples, 0.2, 0.4, 0.6, 0.8, 1 and $2 \mu \mathrm{g}$ of purified PirXI were loaded on the same gel as references. An image of the gel was taken and analyzed using the image processing program Image J (https://imagej.net). The intensity of the bands corresponding to PirXI was quantified and the expression levels were calculated.

\section{Purification of PirXI expressed in S. cerevisiae for crystallography}

A single colony of DS75543 cells containing PirXI was inoculated in $5 \mathrm{ml}$ glucose medium and grown overnight. Next, cultures were diluted into a $50 \mathrm{ml}$ glucose medium to an $\mathrm{OD}_{600}$ of 0.1 and cultivated at $30{ }^{\circ} \mathrm{C}$ until mid-exponential phase. The cells were collected by centrifugation, washed and diluted into a 21 xylose medium to a starting $\mathrm{OD}_{600}$ of 0.1 . The cultures were grown at $30{ }^{\circ} \mathrm{C}$ and harvested when the $\mathrm{OD}_{600}$ reached 3-4. In order to minimize metal contamination and preserve in vivo enzyme-bound metals, we applied a gentle cell lysis method and a minimal purification step required as described below. Buffers used are as follows: A, $10 \mathrm{mM}$ MOPS, $\mathrm{pH} 7.5 ; \mathrm{B}, 10 \mathrm{mM}$ MOPS, pH 7.5+5 mM DTT; C, $10 \mathrm{mM}$ MOPS, $\mathrm{pH}$ $7.5+0.5 \mathrm{M}$ sucrose; $\mathrm{D}, 10 \mathrm{mM}$ MOPS, $\mathrm{pH} 7.5+0.5 \mathrm{M}$ sucrose $+100 \mathrm{U} / g$ cells (w.w.) of zymolyase + EDTAfree protease inhibitor cocktail tablets (Roche); and E, $10 \mathrm{mM}$ MOPS, $\mathrm{pH} 7.5+0.5 \mathrm{M} \mathrm{KCl}$. The harvested cells were washed with buffer $\mathrm{A}$ and the pellets were resuspended in buffer $B$ and subsequently incubated for $10 \mathrm{~min}$ on ice. The cells were centrifuged and washed with buffer $C$. The pellets were resuspended in $25 \mathrm{ml}$ of buffer $\mathrm{D}$ and incubated at $30^{\circ}$ while gently shaken at $50 \mathrm{rpm}$ for $1 \mathrm{~h}$. The disruption of the cell walls of the yeast was monitored under a light microscope. Next, $25 \mathrm{ml}$ of buffer A was added to the cell lysate mixtures and the cells were spun down at $1500 \mathrm{~g}$ for $5 \mathrm{~min}$. The supernatants were carefully collected and centrifuged at $16,500 \mathrm{rpm}$ for $30 \mathrm{~min}$. PirXIs were purified from the CFE using an anion exchange column (Resource Q) by applying an ionic strength gradient using buffer $\mathrm{A}$ and buffer E. Prior to sample loading the CFEs were diluted appropriately to reduce the ionic strength of the samples to a similar level of buffer A. Most of PirXI eluted with $50-100 \mathrm{mM} \mathrm{KCl}$ and the fractions of highest purity were collected for crystallization.

\section{Crystallization and structure determination}

Wild-type xylose isomerase and XI mutant V270AA273G were crystallized by the hanging-drop vapor diffusion method with 14-17\% PEG3350, $0.08 \mathrm{M}$ ammonium sulfate and 0.1 M HEPES, pH 7.0 [22]. For 
Table 4 Data collection and refinement statistics

\begin{tabular}{|c|c|c|}
\hline & Wild type & V270A-A273G \\
\hline Resolution range $(\AA)^{\mathrm{a}}$ & $46.6-1.86(1.89-1.86)$ & $46.6-2.0(2.03-2.00)$ \\
\hline \multicolumn{3}{|l|}{ Cell dimensions } \\
\hline$a, b, c(\AA)$ & $78.5,79.3,91.9$ & $78.6,79.4,92.0$ \\
\hline$a, \beta, \gamma\left(^{\circ}\right)$ & $115.5,90.0,117.1$ & $115.5,90.0,117.1$ \\
\hline $\begin{array}{l}\text { Number of unique reflec- } \\
\text { tions }\end{array}$ & $135,735(5669)$ & $111,563(5325)$ \\
\hline Completeness (\%) & $93.6(79.1)$ & $95.3(92.1)$ \\
\hline Overall I/O (I) & $7.1(1.9)$ & $5.3(2.0)$ \\
\hline$R_{\text {merge }}(\%)$ & $6.7(31.8)$ & $6.8(24.4)$ \\
\hline$R / R_{\text {free }}(\%)$ & 13.9/17.6 & $15.4 / 18.5$ \\
\hline \multicolumn{3}{|c|}{ R.m.s. deviations from ideal values } \\
\hline Bond lengths ( $\AA$ ) & 0.009 & 0.010 \\
\hline Bond angles $\left(^{\circ}\right)$ & 1.55 & 1.55 \\
\hline \multicolumn{3}{|l|}{ Protein residues } \\
\hline $\mathrm{Ca}^{2+}$ ions & $4 \times 2$ & $4 \times 2$ \\
\hline Water molecules & 1907 & 1745 \\
\hline Xylose molecules & 22 & 18 \\
\hline Sulfate ions & 7 & 6 \\
\hline PDB accession ID & $6 \mathrm{~T} 8 \mathrm{E}$ & $6 \mathrm{~T} 8 \mathrm{~F}$ \\
\hline
\end{tabular}

a Values in parentheses are for the highest resolution shell

\section{Funding}

This work was performed within the BE-Basic (project number F01.012) R\&D Program (http://www.be-basic.org/), which is financially supported by an EOS Long Term grant from the Dutch Ministry of Economic Affairs, Agriculture and Innovation (EL\&l).

Availability of data and materials

E. coli expression clones of PirXI mutants reported in this study are available from the corresponding author.

Ethics approval and consent to participate

Not applicable.

\section{Consent for publication}

Not applicable.

\section{Competing interests}

PdW is employed at the DSM Biotechnology Center, part of the company Royal DSM. Royal DSM owns intellectual property rights and commercializes aspects of the technology discussed in this paper. PdW, DBJ, and ML are listed on a patent application on xylose isomerase (WO2018073107).

\section{Author details}

${ }^{1}$ Biochemical Laboratory, Groningen Biomolecular Sciences and Biotechnology Institute, University of Groningen, Nijenborgh 4, 9747 AG Groningen, The Netherlands. ${ }^{2}$ DSM Biotechnology Center, Alexander Fleminglaan 1, 2613 AX Delft, The Netherlands.

Received: 22 October 2019 Accepted: 22 December 2019 Published online: 11 January 2020

\section{References}

1. Briggs KA, Lancashire WE, Hartley BS. Molecular cloning, DNA structure and expression of the Escherichia coli D-xylose isomerase. EMBO J. 1984:3:611-6.

2. Jansen MLA, Bracher JM, Papapetridis I, Verhoeven MD, de Bruijn H, de Waal PP, et al. Saccharomyces cerevisiae strains for second-generation ethanol production: from academic exploration to industrial implementation. FEMS Yeast Res. 2017;17:1-20.

3. Walfridsson M, Anderlund M, Bao X, Hahn-Hägerdal B. Expression of different levels of enzymes from the Pichia stipitis XYL1 and XYL2 genes in Saccharomyces cerevisiae and its effects on product formation during xylose utilisation. Appl Microbiol Biotechnol. 1997;48:218-24.

4. Kötter P, Ciriacy M. Xylose fermentation by Saccharomyces cerevisiae. Appl Microbiol Biotechnol. 1993;38:776-83.

5. Cunha JT, Soares PO, Romaní A, Thevelein JM, Domingues L. Xylose fermentation efficiency of industrial Saccharomyces cerevisiae yeast with separate or combined xylose reductase/xylitol dehydrogenase and xylose isomerase pathways. Biotechnol Biofuels. 2019;12:1-14.

6. Amore R, Wilhelm M, Hollenberg CP. The fermentation of xylose-an analysis of the expression of Bacillus and Actinoplanes xylose isomerase genes in yeast. Appl Microbiol Biotechnol. 1989;30:351-7.

7. Brat D, Boles E, Wiedemann B. Functional expression of a bacterial xylose isomerase in Saccharomyces cerevisiae. Appl Environ Microbiol. 2009:75:2304-11.

8. de Figueiredo Vilela L, de Mello VM, Reis VCB, da Silva Bon EP, Gonçalves Torres FA, Neves BC, et al. Functional expression of Burkholderia cenocepacia xylose isomerase in yeast increases ethanol production from a glucose-xylose blend. Bioresour Technol. 2012;128:792-6.

9. Katahira S, Muramoto N, Moriya S, Nagura R, Tada N, Yasutani N, et al. Screening and evolution of a novel protist xylose isomerase from the termite Reticulitermes speratus for efficient xylose fermentation in Saccharomyces cerevisiae. Biotechnol Biofuels. 2017;10:1-18.

10. Hector RE, Dien BS, Cotta MA, Mertens JA. Growth and fermentation of D-xylose by Saccharomyces cerevisiae expressing a novel D-xylose isomerase originating from the bacterium Prevotella ruminicola TC2-24. Biotechnol Biofuels. 2013;6:84

11. Seike T, Kobayashi Y, Sahara T, Ohgiya S, Kamagata Y. Molecular evolutionary engineering of xylose isomerase to improve its catalytic activity and 
performance of micro-aerobic glucose/xylose co-fermentation in Saccharomyces cerevisiae. Biotechnol Biofuels. 2019;12:1-16.

12. Kuyper M, Harhangi HR, Stave AK, Winkler AA, Jetten MSM, de Laat WTAM, et al. High-level functional expression of a fungal xylose isomerase: the key to efficient ethanolic fermentation of xylose by Saccharomyces cerevisiae? FEMS Yeast Res. 2003;4:69-78.

13. Harhangi HR, Akhmanova AS, Emmens R, van der Drift C, de Laat WTAM, van Dijken JP, et al. Xylose metabolism in the anaerobic fungus Piromyces sp. strain E2 follows the bacterial pathway. Arch Microbiol. 2003;180:134-41.

14. Lee SM, Jellison T, Alper HS. Directed evolution of xylose isomerase for improved xylose catabolism and fermentation in the yeast Saccharomyces cerevisiae. Appl Environ Microbiol. 2012;78:5708-16.

15. Zhou H, Cheng JS, Wang BL, Fink GR, Stephanopoulos G. Xylose isomerase overexpression along with engineering of the pentose phosphate pathway and evolutionary engineering enable rapid xylose utilization and ethanol production by Saccharomyces cerevisiae. Metab Eng. 2012;14:611-22.

16. Kuyper M, Hartog MMP, Toirkens MJ, Almering MJH, Winkler AA, van Dijken JP, et al. Metabolic engineering of a xylose-isomerase-expressing Saccharomyces cerevisiae strain for rapid anaerobic xylose fermentation. FEMS Yeast Res. 2005;5:399-409.

17. Bracher JM, Martinez-Rodriguez OA, Dekker WJC, Verhoeven MD, van Maris AJA, Pronk JT. Reassessment of requirements for anaerobic xylose fermentation by engineered, non-evolved Saccharomyces cerevisiae strains. FEMS Yeast Res. 2019. https://doi.org/10.1093/femsyr/foy104.

18. Kwak S, Jin YS. Production of fuels and chemicals from xylose by engineered Saccharomyces cerevisiae: a review and perspective. Microb Cell Fact. 2017;16:1-15.

19. Hoang PTN, Ko JK, Gong G, Um Y, Lee SM. Genomic and phenotypic characterization of a refactored xylose-utilizing Saccharomyces cerevisiae strain for lignocellulosic biofuel production. Biotechnol Biofuels. 2018;11:1-13.

20. Young E, Lee S, Alper H. Optimizing pentose utilization in yeast: the need for novel tools and approaches. Biotechnol Biofuels. 2010;3:24.

21. Verhoeven MD, Lee M, Kamoen L, Van Den Broek M, Janssen DB, Daran JMG, et al. Mutations in PMR1 stimulate xylose isomerase activity and anaerobic growth on xylose of engineered Saccharomyces cerevisiae by influencing manganese homeostasis. Sci Rep. 2017;7:1-11.

22. Lee M, Rozeboom HJ, de Waal PP, de Jong RM, Dudek HM, Janssen DB. Metal dependence of the xylose isomerase from Piromyces sp. E2 explored by activity profiling and protein crystallography. Biochemistry. 2017:56:5991-6005

23. Cherry JR, Fidantsef AL. Directed evolution of industrial enzymes: an update. Curr Opin Biotechnol. 2003;14:438-43.

24. Packer MS, Liu DR. Methods for the directed evolution of proteins. Nat Rev Genet. 2015;16:379-94.

25. Kuchner O, Arnold FH. Directed evolution of enzyme catalysts. Trends Biotechnol. 1997;15:523-30.

26. de Kreij A, van Burg B, Den Venema G, Vriend G, Eijsink VGH, Nielsen JE. The effects of modifying the surface charge on the catalytic activity of a thermolysin-like protease. J Biol Chem. 2002;277:15432-8.

27. Tawfik DS, Goldsmith M. Directed enzyme evolution: beyond the lowhanging fruit. Curr Opin Struct Biol. 2012;22:406-12.

28. Lutz S. Beyond directed evolution — semi-rational protein engineering and design. Curr Opin Biotechnol. 2011;21:734-43.

29. van Leeuwen JGE, Wijma HJ, Floor RJ, van der Laan JM, Janssen DB. Directed evolution strategies for enantiocomplementary haloalkane dehalogenases: from chemical waste to enantiopure building blocks. ChemBioChem. 2012;13:137-48.

30. Lambeir AM, Lauwereys M, Stanssens P, Mrabet NT, Snauwaert J, Van Tilbeurgh $\mathrm{H}$, et al. Protein engineering of xylose (glucose) isomerase from Actinoplanes missouriensis. 2. Site-directed mutagenesis of the xylose binding site. Biochemistry. 1992;31:5459-66.

31. Kovalevsky AY, Hanson L, Fisher SZ, Mustyakimov M, Mason SA, Trevor Forsyth $\mathrm{V}$, et al. Metal ion roles and the movement of hydrogen during reaction catalyzed by D-xylose isomerase: a joint $X$-ray and neutron diffraction study. Structure. 2010;18:688-99.

32. Guerois R, Nielsen JE, Serrano L. Predicting changes in the stability of proteins and protein complexes: a study of more than 1000 mutations. Mol Biol. 2002;320:369-87.
33. van Leeuwen JGE. Library design and screening strategies for efficient enzyme evolution [Doctoral dissertation]. Groningen: University of Groningen; 2015.

34. Futcher AB, Cox BS. Copy number and the stability of 2-micron circle-based artificial plasmids of Saccharomyces cerevisiae. J Bacteriol. 1984;157:283-90.

35. Kim SR, Skerker JM, Kang W, Lesmana A, Wei N, Arkin AP, et al. Rational and evolutionary engineering approaches uncover a small set of genetic changes efficient for rapid xylose fermentation in Saccharomyces cerevisiae. PLoS ONE. 2013;8:e57048.

36. Papapetridis I, Verhoeven MD, Wiersma SJ, Goudriaan M, van Maris AJA, Pronk JT. Laboratory evolution for forced glucose-xylose co-consumption enables identification of mutations that improve mixed-sugar fermentation by xylose-fermenting Saccharomyces cerevisiae. FEMS Yeast Res. 2018;18:1-17.

37. Coolbear T, Whittaker JM, Daniel RM. The effect of metal ions on the activity and thermostability of the extracellular proteinase from a thermophilic Bacillus, strain EA.1. Biochem J. 1992;287:367-74.

38. Dean KM, Qin Y, Palmer AE. Visualizing metal ions in cells: an overview of analytical techniques, approaches, and probes. Biochim Biophys Acta. 2012;1823:1406-15.

39. Foster AW, Osman D, Robinson NJ. Metal preferences and metallation. J Biol Chem. 2014;289:28095-103.

40. Rangarajan M, Hartley BS. Mechanism of D-fructose isomerization by Arthrobacter D-xylose isomerase. Biochem J. 1992;283:223-33.

41. Collyer CA, Blow DM. Observations of reaction intermediates and the mechanism of aldose-ketose interconversion by D-xylose isomerase. Proc Natl Acad Sci. 1990;87:1362-6.

42. Collyer CA, Henrick K, Blow DM. Mechanism for aldose-ketose interconversion by $\mathrm{D}$-xylose isomerase involving ring opening followed by a 1,2-hydride shift. J Mol Biol. 1990:212:211-35.

43. Kuyper M, Toirkens MJ, Diderich JA, Winkler AA, van Dijken JP, Pronk JT. Evolutionary engineering of mixed-sugar utilization by a xylose-fermenting Saccharomyces cerevisiae strain. FEMS Yeast Res. 2005;5:925-34.

44. Nijland JG, Shin HY, Boender LGM, de Waal PP, Klaassen P, Driessen AJM. Improved xylose metabolism by a CYC8 mutant of Saccharomyces cerevisiae. Appl Environ Microbiol. 2017;83:1-12.

45. Nijland JG, Shin HY, de Jong RM, de Waal PP, Klaassen P, Driessen AJM Engineering of an endogenous hexose transporter into a specific D-xylose transporter facilitates glucose-xylose co-consumption in Saccharomyces cerevisiae. Biotechnol Biofuels. 2014;7:1-11.

46. Milo R, Phillips R. Cell biology by the numbers. 1st ed. New York: Garland Science; 2015.

47. Huang M, Bao J, Hallström BM, Petranovic D, Nielsen J. Efficient protein production by yeast requires global tuning of metabolism. Nat Commun. 2017:8:1131.

48. Osiro KO, Borgström C, Brink DP, Fjölnisdóttir BL, Grauslund MFG. Exploring the xylose paradox in Saccharomyces cerevisiae through in vivo sugar signalomics of targeted deletants. Microb Cell Fact. 2019;18:88.

49. Wasylenko TM, Stephanopoulos G. Metabolomic and ${ }^{13} \mathrm{C}-$ metabolic flux analysis of a xylose- consuming Saccharomyces cerevisiae strain expressing xylose isomerase. Biotechnol Bioeng. 2015;112:470-83.

50. Hasunuma T, Sanda T, Yamada R, Yoshimura K, Ishii J, Kondo A. Metabolic pathway engineering based on metabolomics confers acetic and formic acid tolerance to a recombinant xylose-fermenting strain of Saccharomyces cerevisiae. Microb Cell Fact. 2011;10:2.

51. van Bastelaere PBM, Kersters-hilderson HLM, Lambeirt A. Wild-type and mutant D-xylose isomerase from Actinoplanes missouriensis: metal-ion dissociation constants, kinetic parameters of deuterated and non-deuterated substrates and solvent-isotope effects. Biochem J. 1995:307:135-42.

52. Vieira IPV, Cordeiro GT, Gomes DEB, Melani RD, Vilela LF, Domont GB, et al. Understanding xylose isomerase from Burkholderia cenocepacia: insights into structure and functionality for ethanol production. AMB Express. 2019;9:73.

53. Callens M, Tomme P, Kersters-Hilderson H, Cornelis R, Vangrysperre W, De Bruyne CK. Metal ion binding to D-xylose isomerase from Streptomyces violaceoruber. Biochem J. 1988;250:285-90.

54. van Bastelaere PBM, Vangrysperre W, Kersters-Hildersont $\mathrm{H}$. Kinetic studies of $\mathrm{Mg}^{2+}-\mathrm{Co}^{2+}$ - and $\mathrm{Mn}^{2+}$-activated $\mathrm{D}$-xylose isomerases. Biochem J. 1991;278:285-92. 
55. Dudev T, Lim C. Competition among metal ions for protein binding sites: determinants of metal ion selectivity in proteins. Chem Rev. 2014;114:538-56.

56. Gibson DG, Young L, Chuang RY, Venter JG, Hutchion CA III, Smith HO. Enzymatic assembly of DNA molecules up to several hundred kilobases. Nat Methods. 2009;6:343-5.

57. Gietz RD, Schiestl RH. High-efficiency yeast transformation using the LiAc/SS carrier DNA/PEG method R. Nat Protoc. 2007;2:31-4.

58. Verduyn C, Postma E, Scheffers WA, van Dijken JP. Effect of benzoic acid on metabolic fluxes in yeasts: a continuous-culture study on the regulation of respiration and alcoholic fermentation. Yeast. 1992;8:501-17.

59. Ericsson UB, Hallberg BM, DeTitta GT, Dekker N, Nordlund P. Thermofluorbased high-throughput stability optimization of proteins for structural studies. Anal Biochem. 2006;357:289-98.
60. Kabsch W. XDS. Acta Cryst. 2010;66:125-32.

61. Murshudov GN, Skubák P, Lebedev AA, Pannu NS, Steiner RA, Nicholls RA, et al. REFMAC5 for the refinement of macromolecular crystal structures. Acta Cryst. 2011;67:355-67.

62. Winn MD, Ballard CC, Cowtan KD, Dodson EJ, Emsley P, Evans PR, et al. Overview of the CCP4 suite and current developments. Acta Cryst. 2011;67:235-42.

63. Emsley P, Lohkamp B, Scott WG, Cowtan K. Features and development of Coot. Acta Cryst. 2010;66:486-501.

\section{Publisher's Note}

Springer Nature remains neutral with regard to jurisdictional claims in published maps and institutional affiliations.
Ready to submit your research? Choose BMC and benefit from:

- fast, convenient online submission

- thorough peer review by experienced researchers in your field

- rapid publication on acceptance

- support for research data, including large and complex data types

- gold Open Access which fosters wider collaboration and increased citations

- maximum visibility for your research: over $100 \mathrm{M}$ website views per year

At BMC, research is always in progress.

Learn more biomedcentral.com/submissions 